\title{
ON THE INJECTIVITY OF THE VASSILIEV HOMOMORPHISM OF SINGULAR ARTIN MONOIDS
}

\begin{abstract}
NOELlE ANTONY
I prove general combinatorial properties which apply to singular Artin monoids and examine their relationship with the Vassiliev homomorphism $\eta$. I show that $\eta$ preserves the Intermediate Property, discovered by Corran, which holds in positive singular Artin monoids of finite type. From this it follows that $\eta$ is injective for a class of monoids which include singular Artin monoids of type $I_{2}(p)$, generalising a result of East.
\end{abstract}

\section{INTRODUCTION AND PRELIMINARIES}

We begin with a finite indexing set $I$, and we let $\Gamma^{M}$ be a complete labelled graph with $n$ vertices in one-to-one correspondence with $I$ and with edge labels from the set $\{3,4, \ldots, \infty\}$. For $i \neq j$ let $m_{i j}$ denote the label of the edge between the vertices $i$ and $j$, or set $m_{i j}=2$ if there is no such edge. Put $m_{i i}=1$ for every $i \in I$. Such a graph is known as a Coxeter graph of type $M$ where $M=\left(m_{i j}\right)_{i, j \in I}$ is the associated Coxeter matrix. It is conventional to suppress the edge label whenever $m_{i j}=3$.

Now let $S=\left\{\sigma_{i} \mid i \in I\right\}$, and let $\langle x y\rangle^{q}$ denote the alternating product

$$
\underbrace{x y x \ldots}_{q \text { terms }}
$$

of length $q$. The Artin group of type $M$, denoted $G_{M}$, is the group generated by $S$ subject to the relations

$$
\left\langle\sigma_{i} \sigma_{j}\right\rangle^{m_{i j}}=\left\langle\sigma_{j} \sigma_{i}\right\rangle^{m_{i j}} \quad \text { for } i, j \in I \text { and } m_{i j} \neq \infty
$$

(these are denoted by $\Re_{1}$ and called the braid relations). The Coxeter group of type $M$ is the group generated by $S$ subject to the preceding relations and the relations $\sigma_{i}^{2}=1$ for every $i$ in $I$. In this way we see that Coxeter groups arise as quotient groups of Artin groups. If the Coxeter group of type $M$ is finite then $M$ is said to be of finite type. For a classification of finite Coxeter groups see, for example, [14].

The first, and arguably the most well-known, (non-Abelian) example of an Artin group is the braid group established in [1] by Artin; thus the terminology Artin group is suggested by

Received 17th May, 2004

Supported by an Australian Postgraduate Award. The author is indebted to supervisor David Easdown for his encouragement and for many useful discussions and would also like to recognise the referee's helpful suggestions.

Copyright Clearance Centre, Inc. Serial-fee code: 0004-9727/04 \$A2,00+0.00. 
Brieskorn and Saito in [4]. Observe that $\mathcal{B}_{n+1}$, the braid group on $n+1$ strings, arises from the special case when $I=\{1, \ldots, n\}, m_{i j}=3$ when $|i-j|=1$ and $m_{i j}=2$ when $|i-j| \geqslant 2$. Its associated Coxeter graph is referred to as type $A_{n}$, whilst the corresponding Coxeter group is the symmetric group on $n+1$ letters. When the indexing set $I=\{1,2\}$ and the edge label $m_{12}=p$ for some $p \geqslant 3$, the Coxeter graph is said to be of type $M=I_{2}(p)$.

In their study of knot invariants, Baez [2] and Birman [3] extended the braid group $\mathcal{B}_{n+1}$ by introducing the singular braid monoid on $n+1$ strings, $\mathcal{S} \mathcal{B}_{n+1}$. Analogously Artin groups are extended in [6] and [12] as follows: Put $T=\left\{\tau_{i} \mid i \in I\right\}$ and let $S^{-1}=\left\{\sigma_{i}^{-1} \mid i \in I\right\}$, the set of formal inverses of $S$. The singular Artin monoid of type $M$, denoted $S G_{M}$, is the monoid generated by $S \cup S^{-1} \cup T$ and has as its defining relations $\Re_{1}$, the free group relations $\sigma_{i} \sigma_{i}^{-1}=\sigma_{i}^{-1} \sigma_{i}=1$ and the relations $\mathfrak{R}_{2}$ listed below:

$$
\begin{aligned}
\tau_{i} \tau_{j} & =\tau_{j} \tau_{i} \quad \text { if } m_{i j}=2, \\
\tau_{i}\left\langle\sigma_{j} \sigma_{i}\right\rangle^{m_{i j}-1} & = \begin{cases}\left\langle\sigma_{j} \sigma_{i}\right\rangle^{m_{i j}-1} \tau_{j} & \text { if } m_{i j}<\infty \text { and is odd, or } \\
\left\langle\sigma_{j} \sigma_{i}\right\rangle^{m_{i j}-1} \tau_{i} & \text { if } m_{i j}<\infty \text { and is even, }\end{cases} \\
\tau_{i} \sigma_{i} & =\sigma_{i} \tau_{i} \text { for all } i \text { in } I .
\end{aligned}
$$

REMARK. When the Coxeter graph is of type $A_{n}$, the special case mentioned above, the corresponding singular Artin monoid, $S G_{A_{n}}$, coincides with the singular braid monoid on $n+1$ strings, $\mathcal{S} \mathcal{B}_{n+1}$. It is worth noting here that, although singular Artin monoids are defined (in an abstract sense) by the above generators and relations, $\mathcal{S B}_{n+1}$ was originally introduced geometrically in [2] and [3] and was then shown (in [3, Lemma 3] and [13, a subcase of Theorem 2.1]) to admit the preceding presentation.

Let $\mathfrak{A}$ be a finite set called an alphabet. Elements of $\mathfrak{A}$ are referred to as letters, whilst $\mathfrak{A}^{*}$ denotes the free monoid generated by $\mathfrak{A}$, elements of which are said to be words. In arguments below we may regard a relation formally as an ordered pair of words. If $X$ is a set of ordered pairs of words then $X^{\Sigma}=\{(U, V) \mid(U, V)$ or $(V, U) \in X\}$.

If $A$ and $B$ are words in the above generators, we write $A \approx B$ if $A$ can be transformed into $B$ by the use of the defining relations of $\mathcal{S} G_{M}$, and write $A=B$ if the two words are equal letter by letter. Whenever $W=x_{1} \ldots x_{t}$ for some $x_{1}, \ldots, x_{t} \in S \cup S^{-1} \cup T, \operatorname{Rev}(W)=x_{t} \ldots x_{1}$.

The positive Artin monoid of type $M, G_{M}^{+}$, is the monoid generated by $S$ subject to the braid relations $\Re_{1}$. We define the positive singular Artin monoid, denoted $\mathcal{S} G_{M}^{+}$, to be the monoid generated by $S \cup T$ and the set $\mathfrak{R}$ of relations comprised of both $\mathfrak{R}_{1}$ and $\mathfrak{R}_{2}$ listed above. Where it does not cause confusion we denote elements of $G_{M}, G_{M}^{+}, \mathcal{S} G_{M}$ and $S G_{M}^{+}$by words which represent them. If $A$ and $B$ are words in the generators from the sets $S$ and $T$, we write $A \sim B$ if $A$ can be transformed into $B$ by the use of $\Re$. The following Theorems 1.1(1) and (2) are proved in [16] and [6] respectively:

\section{TheOREM 1.1.}

(1) If $A, B$ are words over $S$ and $A \approx B$ then $A \sim B$. 
(2) Let $M$ be of finite type. If $A, B$ are words over $S \cup T$ and $A \approx B$ then $A \sim B$.

Thus $G_{M}^{+}$always injects into $G_{M}$, whilst $\mathcal{S} G_{M}^{+}$embeds into $S G_{M}$ whenever $M$ is of finite type.

We denote by $\ell(A)$ the length of any word $A$. It is easy to see, by inspection of the set $\Re$ of defining relations, that the following property holds in $\mathcal{S} G_{M}^{+}$:

Whenever $U, V$ are over $S \cup T$ and $U \sim V, \ell(U)=\ell(V)$.

We call this property, in accordance with [6, p. 258], homogeneity. Thus the length of an element is defined to be the length of any word representing it. The reduction property is defined as in [6, p. 258]. By [6, Lemma 15], the cancellation law and the reduction property hold in $S G_{M}^{+}$. By reduction we mean an application of the reduction property.

Let $A$ and $B$ be words in $(S \cup T)^{*}$. We say $A$ (left) divides $B$ or $B$ is a (left) multiple of $A$ if there exists a word $X$ in $\mathcal{S} G_{M}^{+}$such that $B \sim A X$, in which case we write $A \prec B$. We say $A$ right divides $B$ or $B$ is a right multiple of $A$ if there is a word $X$ in $S G_{M}^{+}$such that $B \sim X A$, in which case we write $B \succ A$.

Let $\Omega=\left\{A_{1}, A_{2}, \ldots, A_{k}\right\}$ be a set of words in $(S \cup T)^{*}$. If $\Omega$ has a common multiple then by [6, Corollary 13], $\Omega$ has a least common multiple (unique up to equivalence under $\sim$ ) which we denote by $\operatorname{lcm}\left(A_{1}, A_{2}, \ldots, A_{k}\right)$ or $\operatorname{lcm}(\Omega)$. By homogeneity, $\operatorname{lcm}(\Omega)$ when it exists has minimal length. If $\Omega$ has no common multiple then we write $\operatorname{lcm}\left(A_{1}, A_{2}, \ldots, A_{k}\right)=\infty$.

In Section 2 we discover properties pertaining to fundamental elements in addition to general results regarding divisibility in $\mathcal{S} G_{M}^{+}$. In Section 3 the Vassiliev homomorphism $\eta$ is defined, we state Birman's conjecture and show that if Birman's conjecture is true for $\mathcal{S} G_{M}^{+}$, where $M$ is of finite type, then it is true for $S G_{M}$; this is followed by some observations regarding $\eta$. The results of Sections 4 and 5 hold for finite type $M$. In Section 4 we study the relationship between divisibility in $\mathcal{S} G_{M}^{+}$and the support of $\eta$. In particular, we show that if $U$, $V \in S G_{M}^{+}, C \in G_{M}^{+}$and $\eta(U)=\eta(V)$ then $C$ divides $U$ if and only if $C$ divides $V$. Finally, in Section 5 we prove that $\eta$ preserves the Intermediate Property which holds in $\mathcal{S} G_{M}^{+}$; namely, if $\eta\left(\tau_{i} U\right)=\eta\left(\tau_{j} V\right)$ then $m_{i j} \leqslant 2$. From this it follows that $\eta$ is injective for a class of monoids which include singular Artin monoids of type $I_{2}(p)$, generalising [9, Theorem 14].

\section{THE FUNDAMENTAL WORD $\Delta$}

The following refers to a construction developed in [6, Section 2]. For every generator $\alpha$ and word $W$ in $(S \cup T)^{*}$, the word $K_{\alpha}(W)$ is defined and begins with $\alpha$ if and only if $W$ is divisible by $\alpha$, in which case we write $W_{\alpha}=(W / \alpha)$ for the word obtained by removing the letter $\alpha$ from $K_{\alpha}(W)$. Then the word $(W / V)$ is defined recursively and exists precisely when $V \prec W$ and has the property that $W \sim V(W / V)$.

2.1. Properties of $\Delta$. Suppose in this subsection that $M$ is of finite type. Let $\Delta=\operatorname{lcm}(S)$. We call $\Delta$, in accordance with [11, Section 2], the fundamental word of $S G_{M}$ and write 
$\zeta=\Delta^{2}$. In [4, Theorem 5.6] tells us that $\Delta$ exists precisely when $M$ is of finite type, whilst by [6, Section 5], the following holds:

THEOREM 2.1. Let $T_{1} \subseteq T$, and let $W$ be a word over $S \cup S^{-1} \cup T_{1}$. Then there exists an integer $p$ and a word $\bar{W}$ over $S \cup T_{1}$ such that $W \approx \Delta^{p} \bar{W}$.

In [6, Section 4], Corran showed that there exists a uniquely determined involutionary automorphism of $\mathcal{S} G_{M}$, which we denote by $\mathcal{R}$, with the following property:

1. $\mathcal{R}$ sends letters to letters, so that for any $i \in I, \alpha=\sigma$ or $\tau, \mathcal{R}\left(\alpha_{i}\right)=\alpha_{\phi(i)}$ and $\sigma_{i} \Delta \sim \Delta \mathcal{R}\left(\sigma_{i}\right)$. Hence $\mathcal{R}$ arises from a permutation $\phi$ of $I$ with $\phi^{2}=$ id and $m_{\phi(i) \phi(j)}=m_{i j}$.

(See also [4, Lemma 5.2]). We write $\alpha_{i}^{\prime}=\alpha_{i^{\prime}}$ for $\mathcal{R}\left(\alpha_{i}\right)$. By [6, Lemma 18], we have the ensuing property of $\Delta$ :

LEMMA 2.1. Let $W$ be any word in $(S \cup T)^{*}$. Then $W \Delta \sim \Delta \mathcal{R}(W)$. In particular, $W$ is left divisible by $\Delta$ if and only if $W$ is right divisible by $\Delta$.

The previous result tells us that $\Delta$ acts almost like a central element of $\mathcal{S} G_{M}^{+}$, but not quite, as Lemma 2.2 below shows. The first part of the lemma is a restatement of $[4$, Lemmas 5.2(ii) and 5.1(ii)]; the second part of the result is a combination of [6, Lemma 18] and [4, Theorem 7.2]. All the undefined notation in Lemma 2.2(2) comes from the cited references.

LEMMA 2.2 .

(1) $\mathcal{R}(\Delta) \sim \Delta$ and $\operatorname{Rev}(\Delta) \sim \Delta$.

(2) The centre of the singular Artin monoid is generated by the fundamental element $\Delta$ if the associated involution $\mathcal{R}$ is trivial. The involution $\mathcal{R}$ is non-trivial only for types $A_{n}$ (when $n \geqslant 2$ ), $D_{2 k+1}, E_{6}$ and $I_{2}(2 q+1)$, in which case $\Delta^{2}$ represents the generator of the centre.

Since $\Delta$ is the lowest common multiple of the set $S$, the word $K_{a}(\Delta)$ is defined, and so $\Delta_{a}$ exists for every $a \in S$. By recalling that $\zeta=\Delta^{2}$, we analogously obtain the existence of the word $\zeta_{a}$ for every $a \in S$.

LEMMA 2.3.

(1) For any $a$ in $S, \mathcal{R}\left(\Delta_{a}\right) \sim \Delta_{a^{\prime}}$.

(2) For $\alpha=\sigma$ or $\tau$ and $i \in I, \alpha_{i} \Delta_{\sigma_{i}} \sim \Delta_{\sigma_{i}} \alpha_{i}{ }^{\prime}$. In particular, $a \Delta_{a} \sim \Delta \sim \Delta_{a} a^{\prime}$ whenever $a \in S$.

PROOF: (1) Let $a$ be a generator in $S$. By Lemma 2.2(1) and since $\Delta \sim b \Delta_{b}$ for any $b$ in $S$, it follows that

$$
a^{\prime} \mathcal{R}\left(\Delta_{a}\right) \sim \mathcal{R}\left(a \Delta_{a}\right) \sim \mathcal{R}(\Delta) \sim \Delta \sim a^{\prime} \Delta_{a^{\prime}}
$$

Hence $\mathcal{R}\left(\Delta_{a}\right) \sim \Delta_{a^{\prime}}$ by cancellation, as required.

(2) Let $\sigma_{i}$ be any generator in $S$, and let $\alpha_{i}=\sigma_{i}$ or $\tau_{i}$. Then $\alpha_{i} \sigma_{i} \sim \sigma_{i} \alpha_{i}$, and by Lemma 2.1, $\alpha_{i} \Delta \sim \Delta \alpha_{i}{ }^{\prime}$, so

$$
\sigma_{i} \alpha_{i} \Delta_{\sigma_{i}} \sim \alpha_{i} \sigma_{i} \Delta_{\sigma_{i}} \sim \alpha_{i} \Delta \sim \Delta \alpha_{i}^{\prime} \sim \sigma_{i} \Delta_{\sigma_{i}} \alpha_{i}^{\prime}
$$


The result now follows by cancellation.

The reader is referred to [5, Lemma 2.3] for variations of the preceding Lemmas 2.2 and 2.3 .

2.2. Divisibility THEORY. The results of this subsection hold for positive singular Artin monoids of any (not necessarily finite) type. The ensuing definitions are obtained from [6, Section 2]. Let $C$ be a non-empty word and $a, b \in S \cup T$. We say $C$ is a simple a-chain with source $a$ and target $b$ if there is a (non-empty) word $P$ and (possibly empty) word $Q$ such that $(a P, C b Q)$ is a relation in $\mathfrak{R}^{\Sigma}$. We call $C$ an a-chain if $C=C_{1} \ldots C_{k}$ for simple chains $C_{1}, \ldots, C_{k}$ where the source of $C_{1}$ is $a$ and the source of $C_{i+1}$ is the target of $C_{i}$ for $i=1, \ldots, k-1$. In this case, the source and target of $C$ are defined to be the source of $C_{1}$ and the target of $C_{k}$ respectively.

REMARK 1. In $G_{M}^{+}$, if $C$ is a $\sigma_{a}$-chain to $\sigma_{b}$ then $\operatorname{Rev}(C)$ is a $\sigma_{b}$-chain to $\sigma_{a}$. However this does not always hold in $\mathcal{S} G_{M}^{+}$. For example, if $3 \leqslant m_{a b}<\infty$ then $\sigma_{b}$ is a $\tau_{a}$-chain to $\sigma_{a}$, but $\operatorname{Rev}\left(\sigma_{b}\right)=\sigma_{b}$ is a $\sigma_{a}$-chain to $\sigma_{a} \neq \tau_{a}$. Moreover, $\tau_{a} \sigma_{b}$ is a simple $\sigma_{b}$-chain to $\sigma_{a}$, but the target of the non-simple $\sigma_{a}$-chain $\operatorname{Rev}\left(\tau_{a} \sigma_{b}\right)=\sigma_{b} \tau_{a}$ is $\sigma_{a}$, not equal to the source of $\tau_{a} \sigma_{b}$.

Lemmas 2.4, 2.5 and 2.6 below are restatements of [6, Lemmas 3, 5 and 4(2)].

LEMMA 2.4. If $C$ is an $a$-chain to $b$ and $W$ is a common multiple of $a$ and $C$ then $W$ is also a common multiple of $a$ and $C b$. In particular, $a$ does not divide $C$.

LEMMA 2.5. Suppose $a \in S \cup T$, and let $W$ be a word over $S \cup T$ such that $a$ does not divide $W$ but $\operatorname{Icm}(a, W)$ exists. Then either $W$ is empty or there is an a-chain $C$ such that $W \sim C$.

LEMMA 2.6. If $C$ is an a-chain such that $a$ divides $C b$ then $b$ is the target of $C$.

The following two results are also proved in [7, Lemma 6.5].

LEMmA 2.7. If $C$ is an $a$-chain to $b$ and $a$ is an element of $S$ then $b$ also lies in $S$.

Proof: Write $C=C_{1} \ldots C_{k}$ where each $C_{i}$ is simple, and sappose $d$ is the target of $C_{1}$. Then $\left(a P, C_{1} d Q\right) \in \mathfrak{R}^{\Sigma}$ for some generator $d$ and words $P, Q$. If $d \in T$, inspection of the set $\Re$ of defining relations shows that $Q=1$ (since $C_{1} \neq 1$ ) and $a \in T$. Hence $d$ must lie in $S$. If $k=1$ then $b=d$, and we are done. Otherwise, $C_{2} \ldots C_{k}$ is a $d$-chain to $b$ and $d \in S$, so by induction, $b$ must be an element of $S$ as stated.

LEMMA 2.8. If $C$ is a $\sigma_{a}$-chain to $\sigma_{b}$ then $C$ is not right divisible by $\sigma_{b}$.

Proof: Write $C=C_{1} \ldots C_{k}$ where each $C_{i}$ is simple. Since $\sigma_{a}$ clearly lies in $S$, we deduce from Lemma 2.7 that the source of $C_{k}$ is $\sigma_{c}$ for some $c$ in $I$. Hence there exist words $P$, $Q$ in $\mathcal{S} G_{M}^{+}$such that $\left(C_{k} \sigma_{b} P, \sigma_{c} Q\right) \in \mathfrak{R}^{\Sigma}$. Inspection of $\Re$ immediately shows that $C_{k}$ is not right divisible by its target $\sigma_{b}$. If $k=1$ then $C=C_{k} \nsucc \sigma_{b}$, and we are done. So suppose that $k \geqslant 2$, and put $C^{\prime}=C_{1} \ldots C_{k-1}$. By noting that the source of $C_{k}, \sigma_{c}$, is the target of $C_{k-1}$, we see that $C^{\prime}$ is a $\sigma_{a}$-chain to $\sigma_{c}$, and thus by induction, $C^{\prime} \nsucc \sigma_{c}$. Thus

$$
\sigma_{c} \nprec \operatorname{Rev}\left(C^{\prime}\right) \text {. }
$$


Now put $W=\operatorname{Rev}(C)=\operatorname{Rev}\left(C_{k}\right) \operatorname{Rev}\left(C^{\prime}\right)$. We show that $W$ is not divisible by $\sigma_{b}$, so that $C \nsucc \sigma_{b}$ as required. Recall that $C_{k}$ is a simple $\sigma_{c}$-chain to $\sigma_{b}$. If $C_{k}$ is over $S$ then $\operatorname{Rev}\left(C_{k}\right)$ is a $\sigma_{b}$-chain to $\sigma_{c}$; so if $W$ were divisible by $\sigma_{b}, W$ would be a common multiple of $\operatorname{Rev}\left(C_{k}\right)$ and $\sigma_{b}$, thus $\sigma_{c}$ would divide $\operatorname{Rev}\left(C^{\prime}\right)$ (by Lemma 2.4 and cancellation), and this would obviously contradict (2.1). Suppose then that $C_{k}$ is not over $S$. Then either $C_{k}=\tau_{c}$ or $C_{k}=\tau_{d}\left\langle\sigma_{c} \sigma_{d}\right)^{q}$ for some non-negative integer $q$ such that $q \leqslant m_{c d}-2$.

If $C_{k}=\tau_{c}$ then $b=c$ since $C_{k}$ is a $\sigma_{c}$ chain to $\sigma_{b}$; so if $\sigma_{b}=\sigma_{c}$ were to divide $W=\tau_{c} \operatorname{Rev}\left(C^{\prime}\right)$, reduction would show that $\sigma_{c} \prec \operatorname{Rev}\left(C^{\prime}\right)$ which would contradict (2.1). So assume that

$$
C_{k}=\tau_{d}\left\langle\sigma_{c} \sigma_{d}\right\rangle^{q} \quad \text { for some } q \text { such that } 0 \leqslant q \leqslant m_{c d}-2 .
$$

Thus $C_{k}$ is a $\sigma_{c}$-chain to $\sigma_{b}$ where $b=c$ if $q$ is even and $b=d$ if $q$ is odd. Hence

$$
\begin{aligned}
W=\operatorname{Rev}\left(C_{k}\right) \operatorname{Rev}\left(C^{\prime}\right) & \sim \operatorname{Rev}\left(\left\langle\sigma_{c} \sigma_{d}\right\rangle^{q}\right) \tau_{d} \operatorname{Rev}\left(C^{\prime}\right) \\
& = \begin{cases}\left\langle\sigma_{d} \sigma_{c}\right\rangle^{q} \tau_{d} \operatorname{Rev}\left(C^{\prime}\right) & \text { if } q \text { is even, } \\
\left\langle\sigma_{c} \sigma_{d}\right\rangle^{q} \tau_{d} \operatorname{Rev}\left(C^{\prime}\right) & \text { if } q \text { is odd. }\end{cases}
\end{aligned}
$$

Suppose that $\sigma_{b}$ divides $W$. By recalling that $q \leqslant m_{c d}-2$, reduction and cancellation yield a word $R$ such that

$$
\tau_{d} \operatorname{Rev}\left(C^{\prime}\right) \sim\left\langle\sigma_{d} \sigma_{c}\right\rangle^{m_{c d}-q} R=\sigma_{d} \sigma_{c}\left\langle\sigma_{d} \sigma_{c}\right\rangle^{m_{c d}-(q+2)} R .
$$

Another application of reduction tells us that $\tau_{d}$ divides $\sigma_{c}\left\langle\sigma_{d} \sigma_{c}\right\rangle^{m_{c d}-(q+2)} R$, so that yet again by reduction,

$$
\sigma_{c}\left\langle\sigma_{d} \sigma_{c}\right\rangle^{m_{c d}-(q+2)} R \sim\left\langle\sigma_{c} \sigma_{d}\right\rangle^{m_{c d}-1} R^{\prime}
$$

for some word $R^{\prime}$. Thus

$$
\tau_{d} \operatorname{Rev}\left(C^{\prime}\right) \sim \sigma_{d} \sigma_{c}\left\langle\sigma_{d} \sigma_{c}\right\rangle^{m_{c d}-(q+2)} R \sim \sigma_{d}\left\langle\sigma_{c} \sigma_{d}\right\rangle^{m_{c d}-1} R^{\prime} \sim\left\langle\sigma_{c} \sigma_{d}\right\rangle^{m_{c d}} R^{\prime} .
$$

A final application of reduction now shows that $\sigma_{c} \prec \operatorname{Rev}\left(C^{\prime}\right)$ which contradicts (2.1). This implies that $\sigma_{b}$ does not divide $W=\operatorname{Rev}(C)$, thus $C \nsucc \sigma_{b}$ as required. The result now follows by induction.

REMARK 2. The condition in Lemma 2.8 above, for the source of $C$ to be an element of $S$, is necessary. Let $m_{a b}=3$ and put $U=\sigma_{a}^{2} \sigma_{b} \sigma_{a}$. Then $U$ is clearly right divisible by $\sigma_{a}$. Furthermore $U \sim\left(\sigma_{a} \sigma_{b}\right)\left(\sigma_{a}\right)\left(\sigma_{b}\right)$, the latter being a non-simple $\tau_{b}$-chain with target $\sigma_{a}$.

2.3. THE STRUCTURE OF $\Delta$. For the remainder of this section we resume our supposition that $M$ is of finite type. The ensuing Proposition 2.1 provides an important property of the fundamental word. It was originally discovered for the positive braid monoid in [11, Theorem 8] and was generalised in [4, Lemma 5.3] to show that it holds for positive Artin monoids of finite type. As a preliminary result, we deduce the following: 
LEMma 2.9. Let $a \in S$, and let $W$ be a non-empty word in $(S \cup T)^{*}$. Then $a \nprec W$ if and only if $W \sim C$ for some $a$-chain $C$.

Proof: It is evident that $a$ divides $\Delta$, so by Lemma 2.1, $W \Delta \sim \Delta \mathcal{R}(W)$ is a common multiple of $a$ and $W$; this shows that $\operatorname{lcm}(a, W)$ exists. Thus if $a$ does not divide $W$ then Lemma 2.5 yields the existence of an $a$-chain $C$ such that $W \sim C$. On the other hand, if $W \sim C$ where $C$ is an $a$-chain then $a \nprec C \sim W$ by Lemma 2.4.

PRoposition 2.1. Let $X$ and $Y$ be in $(S \cup T)^{*}$. If $\Delta \prec X Y$ then for every $i \in I$ either $X \succ \sigma_{i}$ or $\sigma_{i} \prec Y$.

Proof: Suppose there exists an $i \in I$ such that $\sigma_{i}$ does not right divide $X$ nor does it divide $Y$. We show $\Delta \nprec X Y$ by induction on $\ell(Y) \geqslant 0$. The result certainly is true if $\ell(X)=0$, whilst if $\ell(Y)=0$ the claim holds by Lemma 2.1. So suppose that both $X$ and $Y$ are non-empty. Since $X \nsucc \sigma_{i}$, we infer from Lemma 2.1 that $X$ cannot be right divisible and so divisible by $\Delta$, the lowest common multiple of the set $S$. Hence $\sigma_{j} \nprec X$ for some $j \in I$. By noting that $X$ is non-empty, Lemma 2.9 may be applied and this yields a $\sigma_{j}$-chain $C$ such that $X \sim C$. By Lemma 2.7, we deduce that the target of $C$ is $\sigma_{k}$ for some $k \in I$, so by Lemma 2.8, $C \nsucc \sigma_{k}$. Hence

$$
C \text { is a } \sigma_{j} \text {-chain to } \sigma_{k}, \quad C \sim X \nsucc \sigma_{i} \text { and } \sigma_{k} .
$$

If $\sigma_{k}$ does not divide $Y$ then by another application of Lemma 2.9, we infer the existence of a $\sigma_{k}$-chain $C^{\prime}$ such that $Y \sim C^{\prime}$, so that $C C^{\prime}$ is a $\sigma_{j}$-chain by (2.2); this implies, by Lemma 2.4, that $\sigma_{j}$, and hence $\Delta$, cannot divide $C C^{\prime} \sim X Y$. So suppose that $\sigma_{k} \prec Y$. Then $i \neq k$ since $\sigma_{i} \nprec Y$. Thus there exists a largest integer $q$ and word $Y^{\prime}$ such that

$$
Y \sim\left\langle\sigma_{k} \sigma_{i}\right\rangle^{q} Y^{\prime} \text { and } \sigma_{d} \nprec Y^{\prime}
$$

where $d=k$ if $q$ is even and $d=i$ if $q$ is odd. Since $\left\langle\sigma_{k} \sigma_{i}\right\rangle^{m_{i k}} \sim\left\langle\sigma_{i} \sigma_{k}\right\rangle^{m_{i k}}$ and $\sigma_{i} \nprec Y$, we have that $1 \leqslant q \leqslant m_{i k}-1$. Put $X^{\prime}=X\left\langle\sigma_{k} \sigma_{i}\right\rangle^{q}$. Then

$$
X Y \sim X\left\langle\sigma_{k} \sigma_{i}\right\rangle^{q} Y^{\prime}=X^{\prime} Y^{\prime}
$$

and $\ell\left(Y^{\prime}\right)<\ell(Y)$. If $\sigma_{d}$ were to right divide $X^{\prime}=X\left\langle\sigma_{k} \sigma_{i}\right\rangle^{q}$, reduction and reversal would yield a word $X^{\prime \prime}$ such that $X\left\langle\sigma_{k} \sigma_{i}\right\rangle^{q} \sim X^{\prime \prime}\left\langle\sigma_{k} \sigma_{i}\right\rangle^{m_{i k}}$; so by noting that $q<m_{i k}, \sigma_{i}$ or $\sigma_{k}$ would right divide $X$ (by cancellation) which would contradict (2.2). Hence $X^{\prime} \nsucc \sigma_{d}$, and by (2.3), $\sigma_{d} \nprec Y^{\prime}$. Since $\ell\left(Y^{\prime}\right)<\ell(Y)$, we deduce by an inductive hypothesis that $\Delta \nprec X^{\prime} Y^{\prime} \sim X Y$ as required. The result now follows by induction.

Corollary 2.1. Let $a$ be in $S$ and $S^{\prime}=S \backslash\{a\}$. Then $\operatorname{lcm}\left(S^{\prime}\right) \prec \Delta_{a}$, so that $\operatorname{lcm}\left(\Delta_{a}, a\right)=\Delta$.

Proof: By the previous proposition applied to $\Delta \sim a \Delta_{a}$, we immediately obtain that $\operatorname{lcm}\left(S^{\prime}\right) \prec \Delta_{a}$. The last statement of the corollary follows since $\Delta=\operatorname{lcm}(S)$ and $\Delta_{a} \prec \Delta$ by Lemma 2.3(2). 
COROLlaRy 2.2. Let $a$ be a letter in $S, U$ a word over $S \cup T, r$ any integer such that $r \geqslant 2$, and suppose $\Delta$ divides $a^{r} U$. Then for any $m$ such that $1 \leqslant m \leqslant r-1, \Delta$ also divides $a^{r-m} U$.

Proof: Put $U^{\prime}=a^{r-m} U$. Then $\Delta$ divides $a^{r} U=a^{m} U^{\prime}$, so by Proposition $2.1, b \prec U^{\prime}$ for every $b$ in $S$ such that $b \neq a$. But $U^{\prime}=a^{r-m} U$ is also divisible by $a$ since $1 \leqslant m \leqslant r-1$, thus $\operatorname{lcm}(S)=\Delta \prec U^{\prime}$ as required.

LEMMA 2.10. Let $I$ and $J$ be words in $(S \cup T)^{*}$ and $a$ an element in $S$. Then the following are equivalent:

(1) $\Delta \prec I \Delta_{a} \Delta_{a^{\prime}} J$;

(2) $\Delta \prec I \Delta_{a}$ or $\Delta \prec \Delta_{a^{\prime}} J$;

(3) $I \succ a$ or $a \prec J$.

In particular, $\left(\Delta_{a} \Delta_{a^{\prime}}\right)^{m}$ is not divisible by $\Delta$ for any $a$ in $S$ and any positive integer $m$.

PRoOF: Suppose $\Delta \prec I \Delta_{a} \Delta_{a^{\prime}} J$. By Proposition 2.1, either $I \succ a$ or $a \prec \Delta_{a} \Delta_{a^{\prime}} J$. If $I \succ a$ then $I \sim I_{1} a$ for some word $I_{1}$ in $(S \cup T)^{*}$, hence $I \Delta_{a} \sim I_{1} a \Delta_{a} \sim I_{1} \Delta$; thus $I \Delta_{a}$ is right divisible and so (by Lemma 2.1) is left divisible by $\Delta$. If $a \prec \Delta_{a} \Delta_{a^{\prime}} J$ then $\Delta \prec \Delta_{a} \Delta_{a^{\prime}} J$ (since $\operatorname{lcm}\left(\Delta_{a}, a\right)=\Delta$ ); by Lemma 2.3(2) and cancellation, we then obtain that $a^{\prime} \prec \Delta_{a^{\prime}} J$ which gives $\Delta \prec \Delta_{a^{\prime}} J$. Hence (2) follows from (1).

Now assume that $\Delta$ divides $I \Delta_{a}$ or $\Delta_{a^{\prime}} J$. If $\Delta \prec I \Delta_{a}$ then $\Delta$ right divides $I \Delta_{a}$ by Lemma 2.1, hence there is a word $I_{1}$ in $(S \cup T)^{*}$ such that $I \Delta_{a} \sim I_{1} \Delta \sim I_{1} a \Delta_{a}$, so by cancellation, $I \succ a$. That $\Delta \prec \Delta_{a^{\prime}} J$ implies $a \prec J$ is deduced immediately by Lemma 2.3(2) and cancellation. Hence (3) follows from (2). That (3) implies (1) is inferred from Lemmas 2.3(2) and 2.1. Observe that (3) yields $\ell(I)$ or $\ell(J)$ is at least 1 ; thus by a simple induction on $m$, the last statement of the lemma is proved.

\section{BIRMAN'S CONJECTURE}

Except when explicitly stated, we assume throughout this section that $M$ is of any type. The reader is reminded that $\mathcal{S B}_{n+1}$ refers to the singular braid monoid on $n+1$ strings, whilst $\mathcal{B}_{n+1}$ denotes the braid group on $n+1$ strings. Moreover, $\mathcal{S} \mathcal{B}_{n+1}$ is precisely the singular Artin monoid of type $A_{n}$ mentioned in the introduction. The map $\eta$ from $S \mathcal{B}_{n+1}$ to the group algebra $\mathbb{Z} \mathcal{B}_{n+1}$, induced by

$$
\sigma_{i}^{ \pm 1} \mapsto \sigma_{i}^{ \pm 1}, \quad \tau_{i} \mapsto \sigma_{i}-\sigma_{i}^{-1} \text { for } i \in I,
$$

is easily proved to be a monoid homomorphism; $\eta$ is sometimes referred to as the Vassiliev homomorphism [18] or desingularisation map [17]. In [3, Remark 1], Birman conjectured that $\eta$ is faithful, so that the singular braid monoid embeds into the group algebra of the braid group. In 1996, Fenn, Rolfsen and Zhu [10] showed that the above map is injective on the set comprised of singular braids with up to two singularities (where a singularity is denoted by a $\tau_{i}$ ); the following year, Zhu [19] extended this result by showing that it holds for up to 
three singular points. Dasbach and Gemein [8], simultaneously but independently of Járai [15], discovered that the conjecture holds for the singular braid monoid on three strings. The conjecture was proved in its entirety by Paris [17], whilst East [9] demonstrated that it holds for all singular Artin monoids of type $I_{2}(p)$. Godelle and Paris later proved the truth of the conjecture for right-angled singular Artin monoids in [12]. In effect, Birman's conjecture generalises to arbitrary Artin monoids since the Vassiliev homomorphism, $\eta$, from any singular Artin monoid to the group algebra of the corresponding Artin group is well defined by the above rule. This fact was observed in [6, Remark 25]. Thus we may conjecture the following.

CONJECTURE 1. The Vassiliev homomorphism $\eta: S G_{M} \longrightarrow \mathbb{Z} G_{M}$ is faithful, so that the singular Artin monoid embeds into the group algebra of the Artin group.

We write $X=Y$ if $X$ and $Y$ are equal elements of $\operatorname{Im}(\eta)$, in which case the context of the equality signs should be made clear.

Analogously the map, also denoted by $\eta$, from $\mathcal{S} G_{M}^{+}$to $\mathbb{Z} G_{M}$, induced by $\sigma_{i} \mapsto \sigma_{i}$, $\tau_{i} \mapsto \sigma_{i}-\sigma_{i}^{-1}$, is a monoid homomorphism, again referred to as the Vassiliev homomorphism.

CONJECTURE 2. The Vassiliev homomorphism : $\mathcal{S} G_{M}^{+} \rightarrow \mathbb{Z} G_{M}$ is injective.

Indeed for finite type $M$, Conjecture 2 implies Conjecture 1 , as the following result demonstrates:

OBSERVATION 1. Whenever $M$ is of finite type, Conjecture 2 implies Conjecture 1.

PROOF: Suppose Conjecture 2 holds and that $\eta(U)=\eta(V)$ for some words $U$ and $V$ in $S G_{M}$ where, without causing confusion, we denote the equivalence class of a word by the word itself. By Theorem 2.1, there are integers $p(U), p(V)$ and words $\bar{U}, \bar{V}$ in $\mathcal{S} G_{M}^{+}$such that $U \approx \Delta^{p(U)} \bar{U}$ and $V \approx \Delta^{p(V)} \bar{V}$. Then there exist positive integers $k_{1}, k_{2}$ and $k$ such that

$$
\Delta^{k} U \approx \Delta^{k_{1}} \bar{U} \text { and } \Delta^{k} V \approx \Delta^{k_{2}} \bar{V} .
$$

By recalling that $\Delta$ is over $S$, we thus deduce that

$$
\eta\left(\Delta^{k_{1}} \bar{U}\right)=\eta\left(\Delta^{k} U\right)=\eta\left(\Delta^{k} V\right)=\eta\left(\Delta^{k_{2}} \bar{V}\right) .
$$

But $k_{1}$ and $k_{2}$ are positive integers, whilst $\bar{U}$ and $\bar{V}$ are over $S \cup T$, so that $\Delta^{k_{1}} \bar{U}$ and $\Delta^{k_{2}} \bar{V}$ also represent elements of $S G_{M}^{+}$, and their images under $\eta$ are the same, in either interpretation of $\eta$. Hence since Conjecture 2 holds, $\Delta^{k_{1}} \bar{U} \sim \Delta^{k_{2}} \bar{V}$. By (3.1) we infer that $\Delta^{k} U \approx \Delta^{k} V$. The result now follows by cancellation.

Observation 1 thus shows that, when $M$ is of finite type, it is sufficient to prove Birman's conjecture in the positive singular Artin monoid. Many properties hold only in $\mathcal{S} G_{M}^{+}$and not in $S G_{M}$; the most obvious such property is preservation of word length, which allows for inductive arguments. In what follows we make some elementary observations about the Vassiliev homomorphism and its relationship with $\mathcal{S} G_{M}^{+}$.

Define monoid homomorphisms $\varepsilon$ and $\mathcal{N}$ from $\mathcal{S} G_{M}$ to $(\mathbb{Z},+)$ by

$$
\varepsilon:{\sigma_{i}}^{ \pm 1} \mapsto \pm 1, \tau_{i} \mapsto 0, \quad \mathcal{N}: \sigma_{i}^{ \pm 1} \mapsto 0, \tau_{i} \mapsto 1
$$


and a map ${ }^{+}$from $(S \cup T)^{*}$ to $S^{*}$ by ${ }^{+}: \alpha_{i} \mapsto \sigma_{i}$, where $\alpha=\sigma$ or $\tau$. So $\varepsilon(A)$ is the exponent sum of sigmas in any word $A$ in $S G_{M} ; \mathcal{N}(A)$ counts the number of singularities (taus) of any word in $S G_{M}$; and + turns every letter from $T$ into a corresponding one in $S$. Moreover, + induces a homomorphism : $S G_{M}^{+} \rightarrow G_{M}^{+}$, and for any word $A$ in $S G_{M}^{+}, \varepsilon\left(A^{+}\right)=\varepsilon(A)$ $+\mathcal{N}(A)$. In $[15$, Lemma 1], Járai showed that we can replace $\eta$ with a simpler homomorphism $\psi$ introduced below and that the group algebra $\mathbb{Z} \mathcal{B}_{n+1}$ contains no zero divisors. Lemma 3.1 below is obtained by replacing $\mathcal{S B} \mathcal{B}_{n+1}$ with $\mathcal{S} G_{M}$ in [15, proof of Lemma 1].

Lemma 3.1. Define the homomorphism $\psi: S G_{M} \rightarrow \mathbb{Z} G_{M}$ by $\psi\left(\tau_{i}\right)=\sigma_{i}+\sigma_{i}^{-1}$ and $\psi\left(\sigma_{i}{ }^{ \pm 1}\right)=\sigma_{i}{ }^{ \pm 1}$. Then for any words $C, A$ and $B$ in $S G_{M}$ :

(1) $\eta(A)=\eta(B)$ if and only if $\psi(A)=\psi(B)$, and

(2) $\psi(C A)=\psi(C B) \Longleftrightarrow \psi(A)=\psi(B) \Longleftrightarrow \psi(A C)=\psi(B C)$.

We define the homomorphism $\psi: \mathcal{S} G_{M}^{+} \rightarrow \mathbb{Z} G_{M}$ as in Lemma 3.1. From the above definitions and result, we deduce the following:

LEMMA 3.2. Let $U$ and $V$ be in $S G_{M}^{+}$, and suppose $\psi(U)=\psi(V)$. Then $\ell(U)$ $=\ell(V)$ and $\mathcal{N}(U)=\mathcal{N}(V)$.

Proof: Observe that $U^{+} \approx V^{+}$since they both represent the unique monomial of maximal exponent sum of $\psi(U)=\psi(V)$. But

$$
\ell(U)=\varepsilon(U)+\mathcal{N}(U)=\varepsilon\left(U^{+}\right)=\varepsilon\left(V^{+}\right)=\varepsilon(V)+\mathcal{N}(V)=\ell(V) .
$$

Notice that for every word $A$ in $S G_{M}$ there is a unique monomial, represented by $A^{-}$, obtained by replacing each $\tau_{i}$ by $\sigma_{i}{ }^{-1}$ in the support of $\psi(A)$, with minimal exponent $\operatorname{sum} \varepsilon(A)-\mathcal{N}(A)$. Then since $\psi(U)=\psi(V)$, it follows that $U^{-} \approx V^{-}$, so

$$
\varepsilon(U)-\mathcal{N}(U)=\varepsilon\left(U^{-}\right)=\varepsilon\left(V^{-}\right)=\varepsilon(V)-\mathcal{N}(V) .
$$

By (3.2) and (3.3), $\mathcal{N}(U)=\mathcal{N}(V)$.

\section{COMMON DIVISORS AND THE VASSILIEV HOMOMORPHISM}

For the remainder of this paper we resume our assumption that $M$ is of finite type. In this section we provide a criterion (expressed in Corollary 4.2 below) for determining when two elements of $\mathcal{S} G_{M}^{+}$with the same image under $\psi$ have a non-trivial common divisor.

4.1. THE POSITIVE FORM. The reader is reminded that $\Delta$ is the lowest common multiple of the set $S$, that $\zeta=\Delta^{2}$, and that the words $\zeta_{a}$, for $a \in S$, are defined as in Section 2.1.

Now let $W$ be a word over $S \cup S^{-1} \cup T$. Then there are words $W_{i}$ over $S \cup T$ and generators $\sigma_{a_{i}}^{-1} \in S^{-1}$ such that

$$
W=W_{0} \sigma_{a_{1}}^{-1} W_{1} \sigma_{a_{2}}^{-1} W_{2} \ldots W_{k-1} \sigma_{a_{k}}^{-1} W_{k} .
$$

In accordance with [6, Section 5], we can define maps $\theta_{1}$ and $\theta_{2}$ by

$$
\theta_{1}(W)=W_{0} \zeta_{a_{1}} W_{1} \zeta_{a_{2}} W_{2} \ldots W_{k-1} \zeta_{a_{k}} W_{k} \text { and } \theta_{2}(W)=k .
$$


So $\theta_{1}$ turns $W$ into a word over $S \cup T$ by replacing each letter $\sigma_{a}^{-1}$ from $S^{-1}$ with a corresponding $\zeta_{a}$, whilst $\theta_{2}$ counts the number of occurrences of letters from $S^{-1}$ in $W$. Furthermore, $\theta_{1}$ acts as the identity on $S \cup T$, and for any words $X$ and $Y, \theta_{1}(X Y)=\theta_{1}(X) \theta_{1}(Y)$. Since $\zeta \alpha \sim \alpha \zeta$ for any generator $\alpha$ in $S \cup T$, by centrality, it can be shown (for example, [6, p. 278]) that $\theta_{1}(W) \approx \zeta^{\theta_{2}(W)} W$.

For every $W$ over $S \cup S^{-1} \cup T$ let $q(W)$ be the largest integer such that the word $\left(\theta_{1}(W) / \Delta^{q(W)}\right)$ is defined. Observe that, for any word $W$ and any $a$ in $S, q\left(a^{-1}\right)=1$ and $q(W) \geqslant \theta_{2}(W) \geqslant 0$. This follows from the fact that (by Lemmas 2.1 and 2.3(1)) $\theta_{1}\left(a^{-1}\right)=\zeta_{a} \sim \Delta_{a} \Delta \sim \Delta \Delta_{a^{\prime}}$ and $\alpha \Delta \sim \Delta \alpha^{\prime}$ for every generator $\alpha$ in $S \cup T$. Moreover, by Lemma 2.1 again, $\Delta^{q(X)+q(Y)} \prec \theta_{1}(X) \theta_{1}(Y)=\theta_{1}(X Y)$, we have $q(X Y) \geqslant q(X)+q(Y) \geqslant 0$ for any words $X$ and $Y$ over $S \cup S^{-1} \cup T$. Since $q(W) \geqslant \theta_{2}(W) \geqslant 0$, the word $\left(\theta_{1}(W) / \Delta^{\theta_{2}(W)}\right)$ is always defined and we shall denote it by $N(W)$. Hence $N(W)$ is also a word over $S \cup T$ and $N$ fixes elements of $(S \cup T)^{*}$. Notice that for any words $X$ and $Y$ over $S \cup S^{-1} \cup T$,

$$
\Delta^{\theta_{2}(X Y)} N(X Y) \sim \theta_{1}(X Y)=\theta_{1}(X) \theta_{1}(Y) \sim \Delta^{\theta_{2}(X)} N(X) \Delta^{\theta_{2}(Y)} N(Y) .
$$

Thus, since $\theta_{2}(X Y)=\theta_{2}(X)+\theta_{2}(Y)$, Lemmas 2.1 and 2.2(2) yield

$$
N(X Y) \sim \begin{cases}N(X) N(Y) & \text { if } \theta_{2}(Y) \text { is even, } \\ \mathcal{R}(N(X)) N(Y) & \text { if } \theta_{2}(Y) \text { is odd, }\end{cases}
$$

by cancellation. Since $N(\Delta)=\Delta$, and by Lemma 2.2(1), $\mathcal{R}(\Delta) \sim \Delta$, we obtain immediately that $N(\Delta Y) \sim \Delta N(Y)$ for any $Y$ over $S \cup S^{-1} \cup T$.

We call a word $W$ in $\left(S \cup S^{-1} \cup T\right)^{*}$ minimal if $q(W)=\theta_{2}(W)$. We call a word $W$ over $S \cup T$ prime if it is not divisible by $\Delta$. By recalling that $q(W)$ is the largest integer such that $\Delta^{q(W)}$ divides $\theta_{1}(W)$, we see that if $W$ is minimal then $N(W)=\left(\theta_{1}(W) / \Delta^{q(W)}\right)$ is prime; whereas $\Delta \nprec N(W)=\left(\theta_{1}(W) / \Delta^{\theta_{2}(W)}\right)$ implies that $q(W)=\theta_{2}(W)$. Thus $W$ is minimal if and only if $N(W)$ is prime.

Lemma 4.1. Let $X$ and $Y$ be words over $S \cup S^{-1} \cup T$ such that $X \approx Y$; and let $a, b$ be distinct elements in $S$. Then:

(1) $N(X) \sim N(Y)$ if and only if $\theta_{2}(X)=\theta_{2}(Y)$.

(2) If $X=a^{-1} X_{1}, Y=b^{-1} Y_{1}$ and $N(X) \sim N(Y)$ then $X$ and $Y$ are not minimal.

PROOF: (1) If $\theta_{2}(X)=\theta_{2}(Y)$ then since $X \approx Y$,

$$
\theta_{1}(X) \approx \zeta^{\theta_{2}(X)} X \approx \zeta^{\theta_{2}(Y)} Y \approx \theta_{1}(Y)
$$

when combined with Theorem 1.1(2), this yields $\theta_{1}(X) \sim \theta_{1}(Y)$, so that

$$
N(X)=\left(\theta_{1}(X) / \Delta^{\theta_{2}(X)}\right) \sim\left(\theta_{1}(Y) / \Delta^{\theta_{2}(Y)}\right)=N(Y) .
$$

Now suppose $N(X) \sim N(Y)$, and suppose further, without loss of generality, that $\theta_{2}(X)$ $-\theta_{2}(Y) \geqslant 0$. By multiplying $\left(\theta_{1}(X) / \Delta^{\theta_{2}(X)}\right)=N(X)$ through on the left by $\Delta^{\theta_{2}(X)}$, we 
obtain

$$
\begin{aligned}
\theta_{1}(X) \sim \Delta^{\theta_{2}(X)} N(X) & \sim \Delta^{\theta_{2}(X)} N(Y) \\
& =\Delta^{\theta_{2}(X)-\theta_{2}(Y)} \Delta^{\theta_{2}(Y)}\left(\theta_{1}(Y) / \Delta^{\theta_{2}(Y)}\right) \\
& \approx \Delta^{\theta_{2}(X)-\theta_{2}(Y)} \theta_{1}(Y) \\
& \approx \Delta^{\theta_{2}(X)-\theta_{2}(Y)} \zeta^{\theta_{2}(Y)} Y \\
& \left.\approx \Delta^{\theta_{2}(X)+\theta_{2}(Y)} X \quad \text { (since } X \approx Y, \zeta=\Delta^{2}\right) .
\end{aligned}
$$

Thus $\Delta^{2 \theta_{2}(X)} X=\zeta^{\theta_{2}(X)} X \approx \theta_{1}(X) \approx \Delta^{\theta_{2}(X)+\theta_{2}(Y)} X$, and we have $\Delta^{2 \theta_{2}(X)} \sim \Delta^{\theta_{2}(X)+\theta_{2}(Y)}$ by cancellation and Theorem 1.1(2), so that $\theta_{2}(X)=\theta_{2}(Y)$.

(2) Suppose $X=a^{-1} X_{1}, Y=b^{-1} Y_{1}$ and $N(X) \sim N(Y)$. By (1), $\theta_{2}(X)=\theta_{2}(Y)$ so

$$
1+\theta_{2}\left(X_{1}\right)=\theta_{2}\left(a^{-1} X_{1}\right)=\theta_{2}\left(b^{-1} Y_{1}\right)=1+\theta_{2}\left(Y_{1}\right)
$$

thus $\theta_{2}\left(X_{1}\right)$ is even if and only if $\theta_{2}\left(Y_{1}\right)$ is even. When combined with (4.1), this implies

$$
N\left(a^{-1}\right) N\left(X_{1}\right) \sim N(X) \sim N(Y) \sim N\left(b^{-1}\right) N\left(Y_{1}\right)
$$

if $\theta_{2}\left(X_{1}\right)$ is even, and

$$
\mathcal{R}\left(N\left(a^{-1}\right)\right) N\left(X_{1}\right) \sim N(X) \sim N(Y) \sim \mathcal{R}\left(N\left(b^{-1}\right)\right) N\left(Y_{1}\right)
$$

if $\theta_{2}\left(X_{1}\right)$ is odd. Let $L=\operatorname{lcm}\left(N\left(a^{-1}\right), N\left(b^{-1}\right)\right)$ which exists by either of the preceding chains of equivalences. Then $L \sim \operatorname{lcm}\left(\Delta_{a^{\prime}}, \Delta_{b^{\prime}}\right)$ (see Lemma $4.2(1)$ below), and since $a \neq b$, we infer from Corollary 2.1 that $\Delta \prec L$. Since $\mathcal{R}(\Delta) \sim \Delta$, it follows that $\Delta$ also divides $\mathcal{R}(L)$. Thus $\Delta$ $N(X)$ $\sim N(Y)$, so that $X$ and $Y$ are not minimal.

LEMMA 4.2. For any positive integers $r, s$ and generator $a$ in $S$,

(1) the word $a^{-r}$ is minimal and

$$
N\left(a^{-r}\right) \sim \begin{cases}\left(\Delta_{a} \Delta_{a^{\prime}}\right)^{m} & \text { if } r=2 m, \\ \left(\Delta_{a^{\prime}} \Delta_{a}\right)^{m} \Delta_{a^{\prime}} & \text { if } r=2 m+1 ;\end{cases}
$$

(2) the word $\left(a^{-r} a^{s}\right)$ is not minimal.

PROOF: (1) The claim certainly holds for $r=1=\theta_{2}\left(a^{-1}\right)$ since by Lemmas 2.1 and 2.3(1), we obtain that $\theta_{1}\left(a^{-1}\right)=\zeta_{a} \sim \Delta_{a} \Delta \sim \Delta \Delta_{a^{\prime}}$, so $N\left(a^{-1}\right) \sim \Delta_{a^{\prime}}$ which is clearly prime. For $r=2=\theta_{2}\left(a^{-2}\right)$ we deduce from (4.1) and Lemma 2.3(1) that

$$
N\left(a^{-2}\right) \sim \mathcal{R}\left(N\left(a^{-1}\right)\right) N\left(a^{-1}\right) \sim \Delta_{a} \Delta_{a^{\prime}} .
$$

Thus $N\left(a^{-2}\right) \sim \Delta_{a} \Delta_{a^{\prime}}$ which is prime by Lemma 2.10. So suppose that $r$ is any integer such that $r \geqslant 3$ and that the claim holds for all $l<r$. If $r=2 m$ then

$$
\begin{aligned}
\theta_{1}\left(a^{-r}\right)=\zeta_{a}^{r}=\zeta_{a}^{r-1} \zeta_{a} & \sim \Delta^{2 m-1}\left(\Delta_{a^{\prime}} \Delta_{a}\right)^{m-1} \Delta_{a^{\prime}} \zeta_{a} \\
& \sim \Delta^{2 m-1}\left(\Delta_{a^{\prime}} \Delta_{a}\right)^{m-1} \Delta_{a^{\prime}} \Delta_{a} \Delta \\
& \sim \Delta^{2 m}\left(\Delta_{a} \Delta_{a^{\prime}}\right)^{m-1} \Delta_{a} \Delta_{a^{\prime}} \\
& =\Delta^{2 m}\left(\Delta_{a} \Delta_{a^{\prime}}\right)^{m} .
\end{aligned}
$$


Note that (4.2) was obtained inductively (since $2 m-1<r$ ), and (4.3) holds by Lemmas 2.1 and 2.3(1). Hence $N\left(a^{-r}\right) \sim\left(\Delta_{a} \Delta_{a^{\prime}}\right)^{m}$ and is prime by Lemma 2.10. For $r=2 m+1,(4.1)$ yields

$$
\begin{aligned}
N\left(a^{-r}\right)=N\left(a^{-1} a^{-2 m}\right) & =N\left(a^{-1}\right) N\left(a^{-2 m}\right) \\
& \sim \Delta_{a^{\prime}}\left(\Delta_{a} \Delta_{a^{\prime}}\right)^{m}
\end{aligned}
$$

which is also prime by Lemma 2.10. The result for all $r$ now follows by induction.

(2) Since $\theta_{2}\left(a^{s}\right)=0,(4.1)$ gives $N\left(a^{-r} a^{s}\right) \sim N\left(a^{-r}\right) N\left(a^{s}\right) \sim N\left(a^{-r}\right) a^{s}$, from which we infer, by the first part of this lemma, that

$$
N\left(a^{-r} a^{s}\right) \sim \begin{cases}\left(\Delta_{a} \Delta_{a^{\prime}}\right)^{m} a^{s} & \text { if } r=2 m, \\ \left(\Delta_{a^{\prime}} \Delta_{a}\right)^{m} \Delta_{a^{\prime}} a^{s} & \text { if } r=2 m+1 .\end{cases}
$$

But by Lemma 2.3(2), $\Delta \sim \Delta_{a^{\prime}} a$. Hence $\Delta$ divides $N\left(a^{-r} a^{s}\right)$ by Lemma 2.1. Thus $N\left(a^{-r} a^{s}\right)$ is not prime, and so the word $\left(a^{-r} a^{s}\right)$ cannot be minimal.

4.2. MiNIMAL WORDS AND THE SUPPORT OF $\psi$. Let $U$ be any word over $S \cup T$, also regarded as an element of $S G_{M}^{+}$. A summand of $\psi(U)$ is any word over $S \cup S^{-1}$ obtained by replacing any given instance of $\tau$ by $\sigma$ or $\sigma^{-1}$. The support of $\psi(U)$ is the set of summands of $\psi(U)$. Let $\mathcal{M}_{U}$ denote the summands of $\psi(U)$ that are minimal. For example, in type $A_{2}$, $\psi\left(\tau_{1} \tau_{2} \sigma_{2} \tau_{1}\right)$ has summands $\sigma_{1} \sigma_{2}^{2} \sigma_{1}, \sigma_{1} \sigma_{2}^{2} \sigma_{1}^{-1}, \sigma_{1} \sigma_{2}^{-1} \sigma_{2} \sigma_{1}, \sigma_{1} \sigma_{2}^{-1} \sigma_{2} \sigma_{1}^{-1}, \sigma_{1}^{-1} \sigma_{2}^{2} \sigma_{1}, \sigma_{1}^{-1} \sigma_{2}^{2} \sigma_{1}^{-1}$, $\sigma_{1}^{-1} \sigma_{2}^{-1} \sigma_{2} \sigma_{1}, \sigma_{1}^{-1} \sigma_{2}^{-1} \sigma_{2} \sigma_{1}^{-1}$, and a routine calculation shows

$$
\mathcal{M}_{\tau_{1} \tau_{2} \sigma_{2} \tau_{1}}=\left\{\sigma_{1} \sigma_{2}^{2} \sigma_{1}, \sigma_{1} \sigma_{2}^{2} \sigma_{1}^{-1}, \sigma_{1}^{-1} \sigma_{2}^{2} \sigma_{1}, \sigma_{1}^{-1} \sigma_{2}^{2} \sigma_{1}^{-1}\right\} .
$$

LEMMA 4.3. Let $U, V$ be words in $(S \cup T)^{*}$ also regarded as elements of $\mathcal{S} G_{M}^{+}$such that $\psi(U)=\psi(V)$. For every summand $F$ of $\psi(U)$ there is a corresponding summand $G$ of $\psi(V)$ such that $F \approx G, \theta_{2}(F)=\theta_{2}(G), N(F) \sim N(G)$ and $F \in \mathcal{M}_{U}$ if and only if $G \in \mathcal{M}_{V}$.

Proof: $\quad$ Let $F$ be any element in the support of $\psi(U)$. Since $F$ represents an element of the Artin group $G_{M}$ and $\psi(U)=\psi(V)$, there is an element $G$ in the support of $\psi(V)$ such that $F \approx G$. Thus $F$ and $G$ are equivalent monomials in $G_{M}$, so their exponent sums must be the same; that is, $\varepsilon(F)=\varepsilon(G)$. Since $\varepsilon(A)=\ell(A)-2 \theta_{2}(A)$ for any word $A$ in $\left(S \cup S^{-1}\right)^{*}$, we have $\ell(F)-2 \theta_{2}(F)=\ell(G)-2 \theta_{2}(G)$. By Lemma 3.2, $\ell(F)=\ell(U)=\ell(V)=\ell(G)$. Hence $\theta_{2}(F)=\theta_{2}(G)$ and $F \approx G$, so that $N(F) \sim N(G)$ by Lemma 4.1(1).

Now let $F$ be an element of $\mathcal{M}_{U}$. Then $F$ is a minimal word in the support of $\psi(U)$. By the previous argument we deduce the existence of an element $G$ in the support of $\psi(V)$ such that $F \approx G, \theta_{2}(F)=\theta_{2}(G)$ and $N(F) \sim N(G)$. But $F$ is minimal, so that $N(F) \sim N(G)$ is prime. This shows that $G$ is a minimal word in the support of $\psi(V)$; that is, $G \in \mathcal{M}_{V}$.

Lemma 4.4. Let $U$ be a word over $S \cup T$ that is divisible by $\Delta$. Then $\mathcal{M}_{U}=\emptyset$.

PRoof: Since $\Delta$ divides $U$, there exists a word $U_{1}$ over $S \cup T$ such that $U \sim \Delta U_{1}$. Thus $\psi(U)=\psi\left(\Delta U_{1}\right)=\Delta \psi\left(U_{1}\right)$. Now let $X$ be any summand of $\psi(U)$. Then by Lemma 4.3 , there 
is a word $Y$ in the support of $\psi\left(\Delta U_{1}\right)$ such that $X \approx Y$ and $N(X) \sim N(Y)$. But $Y=\Delta Y_{1}$ for some word $Y_{1}$ in the support of $\psi\left(U_{1}\right)$. Hence,

$$
\Delta \prec \Delta N\left(Y_{1}\right) \sim N\left(\Delta Y_{1}\right)=N(Y) \sim N(X) ;
$$

this implies that $X$ is not minimal and therefore not an element of $\mathcal{M}_{U}$.

Corollary 4.2 below motivates the next proposition.

Proposition 4.1. Let $C$ be a $\sigma_{a}$-chain to $\sigma_{b}$. Then there exists a word $Z \in \mathcal{M}_{C}$ such that $N(Z) \sim C^{\prime}$ where $C^{\prime}$ is a $\sigma_{a}\left[\sigma_{a}^{\prime}\right]$-chain to $\sigma_{b}$ if $\theta_{2}(Z)$ is even [odd].

Proof: By Lemma 2.4, $\sigma_{a}$ does not divide $C$. Write $C=C_{1} \ldots C_{k}$ where each $C_{i}$ is simple. Lemma 2.7 tells us that the target of $C_{1}$ must lie in $S$. So suppose that $\sigma_{c}$ is the target of $C_{1}$. Then there exist words $P, Q$ such that $\left(\sigma_{a} P, C_{1} \sigma_{c} Q\right) \in \mathfrak{R}^{\Sigma}$. If $C_{1}$ is over $S$, we have that $\sigma_{a} \nprec C_{1}=C_{1}^{+}$is a $\sigma_{a}$-chain to $\sigma_{c}, C_{1}^{+} \in \mathcal{M}_{C_{1}}$, and $\theta_{2}\left(C_{1}^{+}\right)=0$ is even. Otherwise, inspection of the set $\mathfrak{R}$ of definig relations shows that either

$$
C_{1}=\tau_{j}\left\langle\sigma_{a} \sigma_{j}\right\rangle^{q} \quad \text { for some } q \text { such that } 0 \leqslant q \leqslant m_{j a}-2
$$

or $C_{1}=\tau_{a}$. Suppose first that $C_{1}=\tau_{j}\left\langle\sigma_{a} \sigma_{j}\right\rangle^{q}$. Then

$$
\sigma_{a} \nprec N\left(C_{1}^{+}\right)=C_{1}^{+}=\sigma_{j}\left\langle\sigma_{a} \sigma_{j}\right\rangle^{q}=\left\langle\sigma_{j} \sigma_{a}\right\rangle^{q+1}
$$

since $q+1<m_{j_{a}}$. Clearly, $C_{1}^{+}$is a summand of $\psi\left(C_{1}\right)$ which (since it is not divisible by $\sigma_{a}$ ) is prime and so must lie in $\mathcal{M}_{C_{1}}$. Moreover, $\theta_{2}\left(C_{1}^{+}\right)=0$ is even, and $C_{1}^{+}$is a simple $\sigma_{a}$-chain to $\sigma_{c}$ (by definition). So assume that $C_{1}=\tau_{a}$. Then the target of $C_{1}$ is $\sigma_{c}=\sigma_{a}$. Put $X=\sigma_{a}^{-1}$ which is obviously a summand of $\psi\left(C_{1}\right)$, and note that $\theta_{2}(X)=1$ is odd. By Lemma 4.2(1), $X$ is minimal and $N(X) \sim \Delta_{\sigma_{a}^{\prime}}$. Corollary 2.1 tells us that $\sigma_{a}^{\prime}$ does not divide $N(X)$, and thus by Lemma $2.9, N(X) \sim D$ for some $\sigma_{a}^{\prime}$-chain $D$. Since

$$
\sigma_{a}^{\prime} \prec \Delta \sim \Delta_{\sigma_{a}} \sigma_{a} \sim D \sigma_{a}
$$

by Lemma $2.3(2)$, the target of $D$ must be $\sigma_{a}=\sigma_{c}$ by Lemma 2.6 . Hence, in all cases, there exists a word $X$ in $\mathcal{M}_{C_{1}}$ such that $N(X) \sim C_{1}^{\prime}$ where

$$
C_{1}^{\prime} \text { is a } \sigma_{a}\left[\sigma_{a}^{\prime}\right] \text {-chain to } \sigma_{c} \text { if } \theta_{2}(X) \text { is even [odd] }
$$

Now if $k=1$ then $C=C_{1}, \sigma_{b}=\sigma_{c}$, and we are done. Otherwise, $C_{2} \ldots C_{k}$ is a $\sigma_{c}$-chain to $\sigma_{b}$, from which we deduce, by induction, a word $Y$ in $\mathcal{M}_{C_{2} \ldots C_{k}}$ such that $N(Y) \sim C_{2}^{\prime}$ where

$$
C_{2}^{\prime} \text { is a } \sigma_{c}\left[\sigma_{c}^{\prime}\right] \text {-chain to } \sigma_{b} \text { if } \theta_{2}(Y) \text { is even [odd]. }
$$

Put $Z=X Y$, and note that it is a summand of $\psi(C)=\psi\left(C_{1} C_{2} \ldots C_{k}\right)$. Then (4.1) gives

$$
N(Z) \sim \begin{cases}C_{1}^{\prime} C_{2}^{\prime} & \text { if } \theta_{2}(Y) \text { is even, } \\ \mathcal{R}\left(C_{1}^{\prime}\right) C_{2}^{\prime} & \text { if } \theta_{2}(Y) \text { is odd }\end{cases}
$$


CASE 1. $\theta_{2}(Y)$ is even.

Put $C^{\prime}=C_{1}^{\prime} C_{2}^{\prime}$ so that $N(Z) \sim C^{\prime}$. Then $C_{2}^{\prime}$ is a $\sigma_{c}$-chain to $\sigma_{b}$ by (4.5). If $\theta_{2}(Z)$ is even then $\theta_{2}(X)$ is also even, so by (4.4), $C_{1}^{\prime}$ is a $\sigma_{a}$-chain to $\sigma_{c}$, thus $C^{\prime}$ is a $\sigma_{a}$-chain to $\sigma_{b}$ and $C^{\prime} \sim N(Z)$. On the other hand, if $\theta_{2}(Z)$ is odd then $\theta_{2}(X)$ is odd, and by (4.4) again, $C_{1}^{\prime}$ is a $\sigma_{a}^{\prime}$-chain to $\sigma_{c}$; this shows that $C^{\prime}$ is a $\sigma_{a}^{\prime}$-chain to $\sigma_{b}$ as required.

CASE 2. $\theta_{2}(Y)$ is odd.

Put $C^{\prime}=\mathcal{R}\left(C_{1}^{\prime}\right) C_{2}^{\prime}$ so that $N(Z) \sim C^{\prime}$. By (4.5), $C_{2}^{\prime}$ is a $\sigma_{c}^{\prime}$-chain to $\sigma_{b}$. By recalling that $\mathcal{R}$ is an involutionary automorphism of $(S \cup T)^{*}$ which preserves the set of relations $\mathfrak{R}$, we deduce from (4.4) that

$$
\mathcal{R}\left(C_{1}^{\prime}\right) \text { is a } \sigma_{a}^{\prime}\left[\sigma_{a}\right] \text {-chain to } \sigma_{c}^{\prime} \text { if } \theta_{2}(X) \text { is even [odd]. }
$$

Thus, if $\theta_{2}(Z)$ is even then $\theta_{2}(X)$ is odd, so by (4.6), $\mathcal{R}\left(C_{1}^{\prime}\right)$ is a $\sigma_{a}$-chain to $\sigma_{c}^{\prime}$; this implies that $C^{\prime}$ is a $\sigma_{a}$-chain to $\sigma_{b}$. On the other hand, if $\theta_{2}(Z)$ is odd then $\theta_{2}(X)$ is even, so by (4.6) again, $\mathcal{R}\left(C_{1}^{\prime}\right)$ is a $\sigma_{a}^{\prime}$-chain to $\sigma_{c}^{\prime}$, and thus $C^{\prime}$ is a $\sigma_{a}^{\prime}$-chain to $\sigma_{b}$.

Cases 1 and 2 both show that $N(Z) \sim C^{\prime}$ where

$$
C^{\prime} \text { is a } \sigma_{a}\left[\sigma_{a}^{\prime}\right] \text {-chain to } \sigma_{b} \text { if } \theta_{2}(Z) \text { is even [odd]. }
$$

Moreover, by Lemma 2.9, $C_{1}^{\prime}$ is prime. Since $Z$ is a minimal element in the support of $\psi(C)$, it must (by definition) lie in $\mathcal{M}_{C}$, and our proof is complete.

COROLLARY 4.1. Let $U$ be a non-empty word in $(S \cup T)^{*}$ and $a$ any generator in $S$. Suppose that $a \nprec U$. Then $\mathcal{M}_{U}$ contains an element $Z$ such that $a\left[a^{\prime}\right] \nprec N(Z)$ if $\theta_{2}(Z)$ is even [odd]. In particular, $\mathcal{M}_{U} \neq \emptyset$ whenever $U$ is prime.

Proof: Since $a \in S$, we deduce from Lemma 2.9 that $U \sim C$ for some $a$-chain $C$. Proposition 4.1 now yields a word $Z$ in $\mathcal{M}_{C}$ such that $N(Z) \sim C^{\prime}$ where $C^{\prime}$ is an $a\left[a^{\prime}\right]$-chain if $\theta_{2}(Z)$ is even [odd]. By Lemma 2.9 again, we infer that $a\left[a^{\prime}\right] \nprec N(Z)$ if $\theta_{2}(Z)$ is even [odd]. Certainly $\psi(U)=\psi(C)$ where $U$ and $C$ are regarded as (the same) elements of $\mathcal{S} G_{M}^{+}$. By Lemma 4.3, there exists $Z^{\prime} \in \mathcal{M}_{U}$ such that $N\left(Z^{\prime}\right) \sim N(Z)$. Hence

$$
a\left[a^{\prime}\right] \nprec N\left(Z^{\prime}\right) \text { if } \theta_{2}\left(Z^{\prime}\right) \text { is even [odd], }
$$

and the result is proved.

CoROLlary 4.2. Let $U, V$ be words in $(S \cup T)^{*}$ also regarded as elements of $S G_{M}^{+}$ such that $\psi(U)=\psi(V)$, and let $C$. be any word over $S$. Then $C$ divides $U$ if and only if $C$ divides $V$.

PROOF: We first prove the 'only if' part of the statement. Suppose first that $U \sim a U_{1}$ for some generator $a$ in $S$ and word $U_{1}$ over $S \cup T$. Put $F=\Delta_{a^{\prime}} U$ and $G=\Delta_{a^{\prime}} V$. Then $\Delta \prec \Delta_{a^{\prime}} a U_{1} \sim F$ by Lemma $2.3(2)$, and $\psi(F)=\Delta_{a^{\prime}} \psi(U)=\Delta_{a^{\prime}} \psi(V)=\psi(G)$ giving a one-one correspondence between the sets $\mathcal{M}_{F}$ and $\mathcal{M}_{G}$ by Lemma 4.3. Since $\Delta$ divides $F$, 
we deduce from Lemma 4.4 that $\mathcal{M}_{F}=\emptyset$; whilst if $G$ is prime, Corollary 4.1 yields $\mathcal{M}_{G} \neq \emptyset$ which contradicts the existence of the bijection between $\mathcal{M}_{F}$ and $\mathcal{M}_{G}$. Hence $\Delta$ also divides $G=\Delta_{a^{\prime}} V$, so by Lemma 2.3(2) and cancellation, $a \prec V$. This proves the result for $\ell(C)=1$, and by noting that it holds trivially for $\ell(C)=0$, starts an induction. So assume that $C$ divides $U$ and $\ell(C) \geqslant 2$. Then there exists a letter $a$ in $S$ and non-empty word $C_{1}$ over $S$ such that $C=C_{1} a$ and $U \sim C_{1} a U_{1}$ for some word $U_{1}$ over $S \cup T$. By induction, we infer the existence of a word $V_{1}$ over $S \cup T$ such that $V \sim C_{1} V_{1}$. Thus $\psi\left(C_{1} a U_{1}\right)=\psi(U)=\psi(V)=\psi\left(C_{1} V_{1}\right)$ which gives $\psi\left(a U_{1}\right)=\psi\left(V_{1}\right)$ (by Lemma 3.1(2)) and shows that $a$ divides $V_{1}$. Hence $C=C_{1} a$ also divides $V \sim C_{1} V_{1}$ as required, and the result for any $\ell(C)$ follows by induction. Swapping the roles of $U$ and $V$ in the preceding argument proves the converse of the result.

\section{THE INTERMEDIATE LEMMA}

In this section we prove that the Intermediate Property - discovered in [6, Intermediate Lemma] and expressed in Lemma 5.1 below - is preserved under the Vassiliev homomorphism. As a corollary we deduce that $\eta$ is injective for a class of monoids which include singular Artin monoids of type $I_{2}(p)$.

LEMMA 5.1. Let $U, V$ be words in $(S \cup T)^{*}$ such that $\tau_{i} U \sim \tau_{j} V$. Then $m_{i j} \leqslant 2$.

The proofs of the ensuing Lemmas 5.2 and 5.3, although technical, are straightforward and lead to Proposition 5.1 below.

LEMMA 5.2. Let $F$ be a minimal word in $\left(S \cup S^{-1} \cup T\right)^{*}$, and let $q$ be any integer such that $q \geqslant 1$. If $\theta_{2}(F)$ is even [odd] then

(1) $\sigma_{s}^{q} F$ is minimal whenever $\sigma_{s}\left[\sigma_{s}{ }^{\prime}\right] \prec N(F)$, and

(2) $\sigma_{s}^{-q} F$ is minimal whenever $\sigma_{s}\left[\sigma_{s}{ }^{\prime}\right] \nprec N(F)$.

Proof: Suppose $F$ is minimal so $N(F)$ is prime.

CASE 1. $\theta_{2}(F)$ is even.

Suppose first that $\sigma_{s}$ divides $N(F)$, so that $N(F) \sim \sigma_{s} F_{1}$ for some word $F_{1}$ over $S \cup T$. Put $F^{\prime}=\sigma_{s}^{q} F$. By noting that $\theta_{2}(F)$ is even, (4.1) gives

$$
N\left(F^{\prime}\right)=N\left(\sigma_{s}^{q} F\right) \sim N\left(\sigma_{s}^{q}\right) N(F) \sim \sigma_{s}^{q} N(F) .
$$

Now $\Delta \nprec N(F) \sim \sigma_{s} F_{1}$, so by Corollary 2.2 , it follows that

$$
\Delta \nprec \sigma_{s}^{q+1} F_{1}=\sigma_{s}^{q} \sigma_{s} F_{1} \sim \sigma_{s}^{q} N(F) \sim N\left(F^{\prime}\right) .
$$

Hence $N\left(F^{\prime}\right)$ is prime, and thus $F^{\prime}=\sigma_{s}^{q} F$ is minimal. Now assume that $\sigma_{s} \nprec N(F)$, and put $F^{\prime}=\sigma_{s}^{-q} F$. Then since $\theta_{2}(F)$ is even, we infer that

$$
N\left(F^{\prime}\right)=N\left(\sigma_{s}^{-q} F\right) \sim N\left(\sigma_{s}^{-q}\right) N(F)
$$


by (4.1) again; thus

$$
N\left(F^{\prime}\right) \sim N\left(\sigma_{s}^{-q}\right) N(F) \sim \begin{cases}\left(\Delta_{\sigma_{s}} \Delta_{\sigma_{s}^{\prime}}\right)^{m} N(F) & \text { if } q=2 m, \\ \left(\Delta_{\sigma_{s}^{\prime}} \Delta_{\sigma_{s}}\right)^{m} \Delta_{\sigma_{s}^{\prime}} N(F) & \text { if } q=2 m+1,\end{cases}
$$

by Lemma 4.2(1). Since $\sigma_{s} \nprec N(F)$, Lemma 2.10 yields that $N\left(F^{\prime}\right)$ is prime, so $F^{\prime}=\sigma_{s}^{-q} F$ is minimal as required.

CASE 2. $\theta_{2}(F)$ is odd.

Assume $F^{\prime}=\sigma_{s}^{ \pm q} F$. By recalling that $\mathcal{R}$ is an involution, we deduce from (4.1) that

$$
N\left(F^{\prime}\right) \sim \mathcal{R}\left(N\left(\sigma_{s}^{ \pm q}\right)\right) N(F) \text {, so } \mathcal{R}\left(N\left(F^{\prime}\right)\right) \sim N\left(\sigma_{s}^{ \pm q}\right) \mathcal{R}(N(F)) .
$$

Since $\mathcal{R}\left(\sigma_{s}\right)=\sigma_{s}^{\prime} \prec N(F)$ if and only if $\sigma_{s} \prec \mathcal{R}(N(F))$ and $\mathcal{R}\left(N\left(F^{\prime}\right)\right)$ is prime if and only if $N\left(F^{\prime}\right)$ is prime, the argument proceeds exactly as that of each alternative in the previous case.

Lemma 5.3. Let $F=\sigma_{s} F_{1}$ be a minimal word in $\left(S \cup S^{-1} \cup T\right)^{*}$ such that $\Delta$ divides $N\left(\sigma_{r} F\right)$. Then $\sigma_{s}^{-1} F_{1}$ is minimal or $m_{r s}=2$.

PROOF: Suppose $m_{r s} \neq 2$. We show that $\sigma_{s}^{-1} F_{1}$ is minimal.

CASE 1. $\theta_{2}\left(F_{1}\right)$ is even.

By (4.1) we obtain that $N(F)=N\left(\sigma_{s} F_{1}\right) \sim \sigma_{s} N\left(F_{1}\right)$. Since $N(F)$ is prime, by assumption, Lemma 2.1 thus shows that $N\left(F_{1}\right)$ is also prime. Now $\Delta$ divides $N\left(\sigma_{r} F\right)=N\left(\sigma_{r} \sigma_{s} F_{1}\right)$, so by (4.1) again,

$$
\Delta \prec N\left(\sigma_{r} \sigma_{s} F_{1}\right) \sim N\left(\sigma_{r} \sigma_{s}\right) N\left(F_{1}\right) \sim \sigma_{r} \sigma_{s} N\left(F_{1}\right) .
$$

By noting that $m_{r s} \neq 2$, it is evident that $\sigma_{s}$ is the only generator which right divides $\sigma_{r} \sigma_{s}$, hence an application of Proposition 2.1 to (5.1) yields that $\sigma_{j} \prec N\left(F_{1}\right)$ for every $j \neq s$. Since $N\left(F_{1}\right)$ is prime, this implies that $\sigma_{s}$ does not divide $N\left(F_{1}\right)$. Hence $\sigma_{s}^{-1} F_{1}$ is minimal by Lemma 5.2(2), as required.

CASE 2. $\theta_{2}\left(F_{1}\right)$ is odd.

By (4.1) we obtain that $N(F)=N\left(\sigma_{s} F_{1}\right) \sim \sigma_{s}^{\prime} N\left(F_{1}\right)$. Since $N(F)$ is prime, by assumption, Lemma 2.1 thus shows that $N\left(F_{1}\right)$ is also prime. Now $\Delta$ divides $N\left(\sigma_{r} F\right)=N\left(\sigma_{r} \sigma_{s} F_{1}\right)$, so by (4.1) again,

$$
\Delta \prec N\left(\sigma_{r} \sigma_{s} F_{1}\right) \sim \mathcal{R}\left(N\left(\sigma_{r} \sigma_{s}\right)\right) N\left(F_{1}\right) \sim \sigma_{r}^{\prime} \sigma_{s}^{\prime} N\left(F_{1}\right) .
$$

By recalling that $m_{r^{\prime} s^{\prime}}=m_{r s} \neq 2$, it is clear that $\sigma_{s^{\prime}}=\sigma_{s}^{\prime}$ is the only generator which right divides $\sigma_{\tau}^{\prime} \sigma_{s}^{\prime}$, hence an application of Proposition 2.1 to (5.2) yields that $\sigma_{j^{\prime}}=\sigma_{j}^{\prime}$ divides $N\left(F_{1}\right)$ for every $j^{\prime} \neq s^{\prime}$. Since $N\left(F_{1}\right)$ is prime, this implies that $\sigma_{s}^{\prime}$ does not divide $N\left(F_{1}\right)$. Hence $\sigma_{s}^{-1} F_{1}$ is minimal by Lemma 5.2(2), as required. 
Proposition 5.1. Let $U=\tau_{i} U_{1}, V=\tau_{j} V_{1}$ be words in $(S \cup T)^{*}$ also regarded as elements of $S G_{M}^{+}$such that $\psi(U)=\psi(V)$. Then $m_{i j} \leqslant 2$.

ProOF: Suppose $U=\tau_{i} U_{1}$ and $V=\tau_{j} V_{1}$ provide a counter-example. That is, $\psi\left(\tau_{i} U_{1}\right)=\psi\left(\tau_{j} V_{1}\right)$ but $m_{i j} \geqslant 3$. Suppose further that this counter-example is minimal with respect to $\ell(U)$, which by Lemma 3.2, is equal to $\ell(V)$. Then $\ell(U) \geqslant 2$, since $\ell(U)=\ell(V)=1$ gives $\sigma_{i}+\sigma_{i}^{-1}=\psi(U)=\psi(V)=\sigma_{j}+\sigma_{j}^{-1}$ which holds precisely when $i=j$. We first show that $V=\tau_{j} V_{1}$ is not divisible by $\sigma_{j}$. Suppose, by way of contradiction, that it is. Reduction yields a word $P$ such that $V_{1} \sim \sigma_{j} P$, and by recalling that $\psi(U)=\psi(V)$, Corollary 4.2 implies that $\sigma_{j}$ also divides $U=\tau_{i} U_{1}$; this gives, by reduction again, a word $Q$ such that $U_{1} \sim\left\langle\sigma_{j} \sigma_{i}\right\rangle^{m_{i j}-1} Q$. Put $C=\left\langle\sigma_{j} \sigma_{i}\right\rangle^{m_{i j}-1}$. Then

$$
U=\tau_{i} U_{1} \sim \tau_{i} C Q \sim C \tau_{d} Q
$$

where $\tau_{d}$ is the target of $C$. By noting that $C$ is over $S$, we deduce, from Corollary 4.2 again, that

$$
C=\sigma_{j}\left\langle\sigma_{i} \sigma_{j}\right\rangle^{m_{i j-2}} \prec V \sim \tau_{j} \sigma_{j} P \sim \sigma_{j} \tau_{j} P .
$$

Since $m_{i j} \geqslant 3$, we infer that $\sigma_{i} \prec \tau_{j} P$ by cancellation, so that $P \sim\left\langle\sigma_{i} \sigma_{j}\right\rangle^{m_{i j}-1} P^{\prime}$ for some word $P^{\prime}$ over $S \cup T$. Thus

$$
\begin{aligned}
V \sim \sigma_{j} \tau_{j} P \sim \sigma_{j} \tau_{j}\left\langle\sigma_{i} \sigma_{j}\right\rangle^{m_{i j}-1} P^{\prime} & \sim \sigma_{j}\left\langle\sigma_{i} \sigma_{j}\right\rangle^{m_{i j}-1} \tau_{c} P^{\prime} \\
& \sim\left\langle\sigma_{j} \sigma_{i}\right\rangle^{m_{i j}-1} \sigma_{d} \tau_{c} P^{\prime} \\
& =C \sigma_{d} \tau_{c} P^{\prime}
\end{aligned}
$$

and since $\{c, d\}=\{i, j\}, m_{c d} \geqslant 3$. When combined with the preceding equivalence, (5.3) gives

$$
\psi\left(C \sigma_{d} \tau_{c} P^{\prime}\right)=\psi(V)=\psi(U)=\psi\left(C \tau_{d} Q\right),
$$

so that $\psi\left(\sigma_{d} \tau_{c} P^{\prime}\right)=\psi\left(\tau_{d} Q\right)$ by Lemma 3.1(2). Another application of Corollary 4.2 shows that $\sigma_{d}$ divides $\tau_{d} Q$ and this yields a word $Q^{\prime}$ such that $Q \sim \sigma_{d} Q^{\prime}$. Hence

$$
\psi\left(\sigma_{d} \tau_{c} P^{\prime}\right)=\psi\left(\tau_{d} Q\right)=\psi\left(\tau_{d} \sigma_{d} Q^{\prime}\right)=\psi\left(\sigma_{d} \tau_{d} Q^{\prime}\right)
$$

so $\psi\left(\tau_{c} P^{\prime}\right)=\psi\left(\tau_{d} Q^{\prime}\right)$ again by Lemma 3.1(2), and $m_{c d} \geqslant 3$. This contradicts the minimality of $\ell(U)=\ell(V)$ (since $\ell(V)>\ell\left(\tau_{c} P^{\prime}\right)$ ). Thus $\sigma_{j} \nprec V$, from which we deduce, by a final application of Corollary 4.2, that $\sigma_{j} \nprec U$. This shows that the latter word is prime, and we have $\mathcal{M}_{U} \neq \emptyset$ by Corollary 4 .1. So let $X$ be an element of $\mathcal{M}_{U}$ such that

$$
\sigma_{j}\left[\sigma_{j}^{\prime}\right] \nprec N(X) \text { if } \theta_{2}(X) \text { is even [odd], }
$$

the existence of which is guaranteed also by Corollary 4.1. Assume further that $\theta_{2}(X)=k$ is maximal; that is, if $G$ is any other word in $\mathcal{M}_{U}$ such that

$$
\sigma_{j}\left[\sigma_{j}^{\prime}\right] \nprec N(G) \text { if } \theta_{2}(G) \text { is even [odd] }
$$


then $\theta_{2}(G) \leqslant k$. Now since $U=\tau_{i} U_{1}$, we deduce that either $X=\sigma_{i}^{-1} X_{1}$ or $X=\sigma_{i} X_{1}$ for some summand $X_{1}$ of $\psi\left(U_{1}\right)$. We consider each case separately and show that each implies a contradiction.

CASE $1 . X=\sigma_{i}^{-1} X_{1}$.

Then $\theta_{2}(X)=1+\theta_{2}\left(X_{1}\right)$, so $\theta_{2}(X)$ is even precisely when $\theta_{2}\left(X_{1}\right)$ is odd. Thus

$$
N(X)=N\left(\sigma_{i}^{-1} X_{1}\right) \sim \begin{cases}N\left(\sigma_{i}^{-1}\right) N\left(X_{1}\right) & \text { if } \theta_{2}\left(X_{1}\right) \text { is even, } \\ \mathcal{R}\left(N\left(\sigma_{i}^{-1}\right)\right) N\left(X_{1}\right) & \text { if } \theta_{2}\left(X_{1}\right) \text { is odd, }\end{cases}
$$

by (4.1), and so

$$
N(X) \sim \begin{cases}\Delta_{\sigma_{i}^{\prime}} N\left(X_{1}\right) & \text { if } \theta_{2}(X) \text { is odd, } \\ \Delta_{\sigma_{i}} N\left(X_{1}\right) & \text { if } \theta_{2}(X) \text { is even, }\end{cases}
$$

by Lemmas $4.2(1)$ and 2.3(1). This implies, by Corollary 2.1, that $\sigma_{j}\left[\sigma_{j}^{\prime}\right] \prec N(X)$ if $\theta_{2}(X)$ is even [odd] which clearly contradicts (5.4).

CASE 2. $X=\sigma_{i} X_{1}$.

Since $\psi(U)=\psi(V)$ and $X \in \mathcal{M}_{U}$, Lemma 4.3 yields the existence of a word $Y$ in $\mathcal{M}_{V}$ such that

$$
N\left(\sigma_{i} X_{1}\right)=N(X) \sim N(Y) \quad \text { and } \quad \theta_{2}(X)=\theta_{2}(Y)=k .
$$

By noting that $V=\tau_{j} V_{1}$, we deduce that $Y=\sigma_{j}^{ \pm 1} Y_{1}$ for some word $Y_{1}$ in the support of $\psi\left(V_{1}\right)$. If $Y=\sigma_{j} Y_{1}$ then $\theta_{2}(X)=\theta_{2}(Y)=\theta_{2}\left(Y_{1}\right)$ by (5.5); this gives

$$
N(X) \sim N\left(\sigma_{j} Y_{1}\right) \sim \begin{cases}\sigma_{j} N\left(Y_{1}\right) & \text { if } \theta_{2}(X) \text { is even, } \\ \sigma_{j}^{\prime} N\left(Y_{1}\right) & \text { if } \theta_{2}(X) \text { is odd, }\end{cases}
$$

by (4.1) and contradicts (5.4). Hence $Y=\sigma_{j}^{-1} Y_{1}$. Observe that the word $\sigma_{j} Y=\sigma_{j} \sigma_{j}^{-1} Y_{1}$ is not minimal by Lemma $4.2(2)$, so (4.1) and (5.5) imply

$$
\begin{aligned}
\Delta \prec N\left(\sigma_{j} Y\right) & \sim \begin{cases}\sigma_{j} N(X) & \text { if } \theta_{2}(X) \text { is even, } \\
\sigma_{j}^{\prime} N(X) & \text { if } \theta_{2}(X) \text { is odd, }\end{cases} \\
& \sim N\left(\sigma_{j} X\right)=N\left(\sigma_{j} \sigma_{i} X_{1}\right) .
\end{aligned}
$$

By recalling that $m_{i j} \geqslant 3$ and that the word $X=\sigma_{i} X_{1}$ is minimal, we deduce from Lemma 5.3 that the word $\sigma_{i}^{-1} X_{1}$ is also minimal. Since $U=\tau_{i} U_{1}$ and $X_{1}$ is a summand of $\psi\left(U_{1}\right)$, this shows that $\sigma_{i}^{-1} X_{1} \in \mathcal{M}_{U}$. Put $F_{1}=\sigma_{i}^{-1} X_{1}$. Then

$$
\theta_{2}\left(F_{1}\right)=1+\theta_{2}\left(X_{1}\right)=1+\theta_{2}\left(\sigma_{i} X_{1}\right)=1+\theta_{2}(X)=1+k
$$


and

$$
N\left(F_{1}\right) \sim \begin{cases}\Delta_{\sigma_{i}^{\prime}} N\left(X_{1}\right) & \text { if } \theta_{2}\left(X_{1}\right) \text { is even, } \\ \Delta_{\sigma_{i}} N\left(X_{1}\right) & \text { if } \theta_{2}\left(X_{1}\right) \text { is odd, }\end{cases}
$$

by (4.1), Lemmas $4.2(1)$ and 2.3(1). Since $F_{1}$ is an element of $\mathcal{M}_{U}$, Lemma 4.3 yields the existence of a word $G_{1}$ in $\mathcal{M}_{V}$ such that $F_{1} \approx G_{1}$,

$$
N\left(F_{1}\right) \sim N\left(G_{1}\right) \text { and } \theta_{2}\left(G_{1}\right)=\theta_{2}\left(F_{1}\right)=1+k .
$$

By noting that $V=\tau_{j} V_{1}$, we deduce that $G_{1}=\sigma_{j}^{ \pm 1} Y_{2}$ for some summand $Y_{2}$ of $\psi\left(V_{1}\right)$. If $G_{1}=\sigma_{j}^{-1} Y_{2}$ then this would contradict that $F_{1}$ is minimal by (5.6) and Lemma 4.1(2). Thus $G_{1}=\sigma_{j} Y_{2}$, so by (5.4),

$$
N\left(\sigma_{i}^{-1} X_{1}\right)=N\left(F_{1}\right) \sim N\left(G_{1}\right)=N\left(\sigma_{j} Y_{2}\right)
$$

and

$$
1+k=\theta_{2}\left(F_{1}\right)=\theta_{2}\left(G_{1}\right)=\theta_{2}\left(\sigma_{j} Y_{2}\right) .
$$

Observe that the word $\sigma_{i} F_{1}=\sigma_{i} \sigma_{i}^{-1} X_{1}$ is not minimal by Lemma 4.2(2), so

$$
\begin{aligned}
\Delta \prec N\left(\sigma_{i} F_{1}\right) & \sim \begin{cases}\sigma_{i} N\left(G_{1}\right) & \text { if } \theta_{2}\left(G_{1}\right) \text { is even, } \\
\sigma_{i}^{\prime} N\left(G_{1}\right) & \text { if } \theta_{2}\left(G_{1}\right) \text { is odd, }\end{cases} \\
& \sim N\left(\sigma_{i} G_{1}\right)=N\left(\sigma_{i} \sigma_{j} Y_{2}\right)
\end{aligned}
$$

by (4.1), (5.7) and (5.8). Since $m_{i j} \geqslant 3$ and the word $G_{1}=\sigma_{j} Y_{2}$ is an element of $\mathcal{M}_{V}$, we deduce from Lemma 5.3 that the word $\sigma_{j}^{-1} Y_{2}$ is also minimal. This shows that $Y_{2}$ must lie in $\mathcal{M}_{V}$ (since $V=\tau_{j} V_{1}$ and $Y_{2}$ is a summand of $\psi\left(V_{1}\right)$ ). Put $G_{2}=\sigma_{j}^{-1} Y_{2}$. Then (5.8) gives

$$
\theta_{2}\left(G_{2}\right)=\theta_{2}\left(\sigma_{j}^{-1} Y_{2}\right)=1+\theta_{2}\left(Y_{2}\right)=2+k,
$$

and since $G_{2}$ is minimal, we obtain

$$
\Delta \nprec N\left(G_{2}\right)=N\left(\sigma_{j}^{-1} Y_{2}\right) \sim \begin{cases}\Delta_{\sigma_{j}^{\prime}} N\left(Y_{2}\right) & \text { if } \theta_{2}\left(Y_{2}\right) \text { is even, } \\ \Delta_{\sigma_{j}} N\left(Y_{2}\right) & \text { if } \theta_{2}\left(Y_{2}\right) \text { is odd, }\end{cases}
$$

by (4.1), Lemmas $4.2(1)$ and 2.3(1). This implies, by Corollary 2.1 , that

$$
\sigma_{j}\left[\sigma_{j}^{\prime}\right] \nprec N\left(G_{2}\right) \text { if } \theta_{2}\left(G_{2}\right) \text { is even [odd] }
$$

since $\theta_{2}\left(G_{2}\right)$ is even if and only if $\theta_{2}\left(Y_{2}\right)$ is odd by (5.9). By recalling that $G_{2} \in \mathcal{M}_{V}$, a final application of Lemma 4.3 yields the existence of a word $F_{2}$ in $\mathcal{M}_{U}$ such that

$$
N\left(F_{2}\right) \sim N\left(G_{2}\right) \text { and } \theta_{2}\left(F_{2}\right)=\theta_{2}\left(G_{2}\right) .
$$


When combined with (5.9) and (5.10), this gives $\theta_{2}\left(F_{2}\right)=2+k>k=\theta_{2}(X)$ and

$$
\sigma_{j}\left[\sigma_{j}^{\prime}\right] \nprec N\left(F_{2}\right) \text { if } \theta_{2}\left(F_{2}\right) \text { is even [odd], }
$$

which contradicts the maximality of $\theta_{2}(X)$ and (5.4).

Recall that $S G_{M}$ denotes the monoid of type $M$ generated by $S \cup S^{-1} \cup T$ where $S=\left\{\sigma_{i} \mid i \in I\right\}, T=\left\{\tau_{i} \mid i \in I\right\}$, and $S^{-1}$ consists of the set of formal inverses of $S$. Recall also that the singular braid monoid on $n+1$ strings, $S \mathcal{B}_{n+1}$, is the singular Artin monoid of type $A_{n}$; the special case obtained when $I=\{1,2, \ldots n\}, m_{i j}=3$ when $|i-j|=1$ and $m_{i j}=2$ whenever $|i-j| \geqslant 2$. The singular Artin monoid of type $I_{2}(p)$ is the special case when $I=\{1,2\}$ and $m_{12}=p \geqslant 3$. Thus if $p=3$, types $A_{2}$ and $I_{2}(3)$ coincide; the singular braid monoid on three strings, $\mathcal{S B}_{3}$, is also the singular Artin monoid of type $I_{2}(3)$. Both types $A_{n}$ and $I_{2}(p)$ are finite (see, for example, [14, Chapter 2]).

For any $i, j \in I$ such that $m_{i j} \geqslant 3$ let $T_{i j}$ denote the monoid generated by $S \cup S^{-1} \cup\left\{\tau_{i}, \tau_{j}\right\}$ subject to the same defining relations as $S G_{M}$. Let $T_{i j}^{+}$denote the set of equivalence classes of words in $\left(S \cup\left\{\tau_{i}, \tau_{j}\right\}\right)^{*}$ under $\sim$. Then $T_{i j}$ and $T_{i j}^{+}$are both submonoids of $\mathcal{S} G_{M}$ and $\mathcal{S} G_{M}^{+}$ respectively.

Proposition 5.2. The restriction of $\eta$ from $T_{i j}$ to the group algebra $\mathbb{Z} G_{M}$ is injective. In particular, the Vassiliev homomorphism $\eta: \mathcal{S} G_{I_{2}(p)} \longrightarrow \mathbb{Z} G_{I}$ is faithful.

PROOF: By Lemma 3.1(1), it suffices to prove the result for $\psi$. We first prove the result for the positive monoid $T_{i j}^{+}$. Suppose that $U, V$ in $\left(S \cup\left\{\tau_{i}, \tau_{j}\right\}\right)^{*}$ provide a counter-example. That is, assume $U \not V$ but $\psi(U)=\psi(V)$. Suppose further that this counter-example is minimal with respect to $\ell(U)$, which by Lemma 3.2, is the same as $\ell(V)$. Clearly $\ell(U) \geqslant 2$. If $U \sim C U^{\prime}, V \sim C V^{\prime}$ for some non-empty word $C$ then $\psi\left(U^{\prime}\right)=\psi\left(V^{\prime}\right)$ by Lemma 3.1(2), $U^{\prime} \nsim V^{\prime}, \ell\left(U^{\prime}\right)<\ell(U)$, and hence the minimality of $\ell(U)$ is contradicted. Thus $U, V$ have no common divisor, from which we infer, by Corollary 4.2 , that $U$ and $V$ are not divisible by any generator from $S$. This tells us that $U=\tau_{r} U_{1}$ and $V=\tau_{s} V_{1}$ for some words $U_{1}, V_{1}$ in $T_{i j}^{+}$and generators $\tau_{r}, \tau_{s} \in\left\{\tau_{i}, \tau_{j}\right\}$. By noting that $m_{i j} \geqslant 3$, we deduce from Proposition 5.1 that $r=s$; this shows that $\tau_{r}$ is a common divisor of $U$ and $V$ and so contradicts that ged $(U, V)=1$. The result thus holds for $T_{i j}^{+}$.

Observe that $\zeta^{-\theta_{2}(W)} \theta_{1}(W) \approx W$ for any word $W$ in $T_{i j}$ and $\theta_{1}(W)$ is an element of $T_{i j}^{+}$. The result for $T_{i j}$ thus follows by an argument identical to that of Observation 1. By putting $I=\{1,2\}$ and $m_{12}=p \geqslant 3$ we obtain $S G_{I_{2}(p)}=T_{12}$, and this proves the second statement of the proposition.

\section{REFERENCES}

[1] E. Artin, 'Theorie der Zöpfe', Hamburg Abh. 4 (1925), 47-72.

[2] J. Baez, 'Link invariants of finite type and perturbation theory', Lett. Math. Phys. 26 (1992), 43-51. 
[3] J.S. Birman, 'New points of view in knot theory', Bull. Amer. Math. Soc. (N.S.) 28 (1993), 253-286.

[4] E. Brieskorn and K. Saito, 'Artin-Gruppen und Coxeter-Gruppen', Invent. Math. 17 (1972), 245-271.

[5] R. Charney, 'Artin groups of finite type are biautomatic', Math. Ann. 292 (1992), 671-683.

[6] R. Corran, 'A normal form for a class of monoids including the singular braid monoid', J. Algebra 223 (2000), 256-282.

[7] R. Corran, On monoids related to braid groups, Ph.D. Thesis (School of Mathematics and Statistics, University of Sydney, 2000).

[8] O.T. Dasbach and B. Gemein, 'A faithful representation of the singular braid monoid on three strands', Ser. Knots Everything 24 (2000), 48-58.

[9] J. East, 'Birman's conjecture is true for $I_{2}(p)$ ', (preprint) http://www.maths.usyd.edu.au:8000/ u/pubs/publist/ preprints/2002/east-8.html.

[10] R. Fenn, D. Rolfsen and J. Zhu, 'Centralizers in the braid group and the singular braid monoid', Enseign. Math. 42 (1996), 75-96.

[11] F.A. Garside, 'On the braid group and other groups', Quart. J. Math. Oxford Ser. (2) 20 (1969), 235-254.

[12] E. Godelle and L. Paris, 'On singular Artin monoids', http://math.u-bourgogne.fr/topolog/ IMB2-publication.html année 2003, n.356.

[13] J. González-Meneses, 'Presentations for the monoids of singular braids on closed surfaces', Comm. Algebra 30 (2002), 2829-2836..

[14] J.E. Humphreys, Reflection groups and Coxeter groups, Cambridge Studies in Advanced Mathematics 29 (Cambridge Univ. Press, Cambridge, U.K., 1990).

[15] A. Járai Jr., 'On the monoid of singular braids', Topology Appl. 96 (1999), 109-119.

[16] L. Paris, 'Artin monoids inject in their groups', Comment. Math. Helv. 77 (2002), 609-637.

[17] L. Paris, 'The proof of Birman's conjecture on singular braid monoids', (preprint) http://math.u-bourgogne.fr/topolog/IMB2-publication.html année 2003, n.338 ArXiv:math.GR/ 0306422 v1.

[18] V. Vassiliev, Cohomology of knot spaces, (V.I. Arnold, Editor), Theory of Singularities and its Applications 1 (Amer. Math. Soc., Profidence, R.I., 1990).

[19] J. Zhu, 'On singular braids', J. Knot Theory Ramifications 6 (1997), 427-440.

School of Mathematics and Statistics

University of Sydney

New South Wales 2006

Australia. 